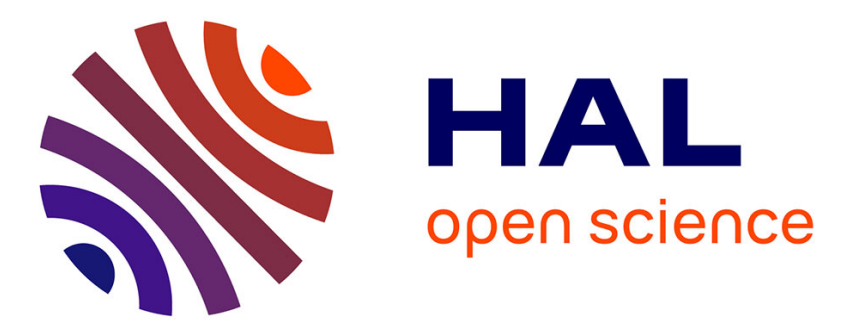

\title{
A cohort longitudinal study identifies morphology and hemodynamics predictors of abdominal aortic aneurysm growth
}

\author{
Florian Joly, Gilles Soulez, Simon Lessard, Claude Kauffmann, Irene \\ Vignon-Clementel
}

\section{To cite this version:}

Florian Joly, Gilles Soulez, Simon Lessard, Claude Kauffmann, Irene Vignon-Clementel. A cohort longitudinal study identifies morphology and hemodynamics predictors of abdominal aortic aneurysm growth. Annals of Biomedical Engineering, In press, 10.1007/s10439-019-02375-1 . hal-02302464

\section{HAL Id: hal-02302464 \\ https://hal.science/hal-02302464}

Submitted on 1 Oct 2019

HAL is a multi-disciplinary open access archive for the deposit and dissemination of scientific research documents, whether they are published or not. The documents may come from teaching and research institutions in France or abroad, or from public or private research centers.
L'archive ouverte pluridisciplinaire HAL, est destinée au dépôt et à la diffusion de documents scientifiques de niveau recherche, publiés ou non, émanant des établissements d'enseignement et de recherche français ou étrangers, des laboratoires publics ou privés. 


\title{
- A cohort longitudinal study identifies morphology and
} 2 hemodynamics predictors of abdominal aortic aneurysm

${ }_{4} \quad$ Florian Joly ${ }^{1,3}$, Gilles Soulez ${ }^{2}$, Simon Lessard ${ }^{2}$, Claude Kauffmann ${ }^{2}$, and Irene Vignon-Clementel ${ }^{1,3}$

affiliations: ${ }^{1}$ Inria, Centre de recherche de Paris, 75012 Paris, France ${ }^{2}$ Department of Medical Imaging, University of Montreal Hospital Research Center, H2X0C1 Montreal, Canada ${ }^{3}$ Sorbonne Université, Laboratoire Jacques-Louis Lions, 75005 Paris, France.

abbreviated title: A cohort longitudinal study identifies AAA risk predictors

corresponding author: I. Vignon-Clementel, Centre de Recherche Inria de Paris, 2 rue Simone Iff, CS 42112, 75589 Paris Cedex 12 (FRANCE)

Phone: +33.1.80.49.42.67

e-mail: irene.vignon-clementel@inria.fr

\begin{abstract}
Abdominal aortic aneurysms (AAA) are localized, commonly occurring aortic dilations. Following rupture only immediate treatment can prevent morbidity and mortality. AAA maximal diameter and growth are the current metrics to evaluate the associated risk and plan intervention. Although these criteria alone lack patient specificity, predicting their evolution would improve clinical decision. If the disease is known
\end{abstract}


to be associated with altered morphology and blood flow, intraluminal thrombus deposit and clinical symptoms, the growth mechanisms are yet to be fully understood. In this retrospective longitudinal study of 138 scans, morphological analysis and blood flow simulations for 32 patients with clinically diagnosed AAAs and several follow-up CT-scans, are performed and compared to 9 control subjects. Several metrics stratify patients between healthy, low and high risk groups. Local correlations between hemodynamic metrics and AAA growth are also explored but due to their high inter-patient variability, do not explain AAA heterogeneous growth. Finally, high-risk predictors trained with successively clinical, morphological, hemodynamic and all data, and their link to the AAA evolution are built from supervise learning. Predictive performance is high for morphological, hemodynamic and all data, in contrast to clinical data. The morphology-based predictor exhibits an interesting effort-predictability tradeoff to be validated for clinical translation.

keywords: abdominal aortic aneurysm; growth; CFD (Computational Fluid Dynamics); haemodynamics; ILT (Intra-Luminal Thrombus); longitudinal study; risk prediction; supervised learning; wall shear stress 


\section{Introduction}

Abdominal aortic aneurysms (AAA) are local dilations of the abdominal aorta which can rupture when blood pressure overcomes artery wall resistance. Following rupture only urgent treatment can prevent morbidity and mortality. It is the $14^{\text {th }}$ leading cause of death in the $\mathrm{USA}^{\frac{57}{57}}$ with a prevalence of $8.9 \%$ for men and $2.2 \%$ for women.

AAA are generally asymptomatic and generally detected through unrelated examinations. Risk is assessed using its maximal diameter $\left(D_{\max }\right)^{\frac{47}{47}}$, taken at the outer wall of the aneurysm on a plane perpendicular to the lumen centerline ${ }^{16}$. It includes the lumen, the Intra Luminal Thrombus (ILT) and the arterial wall which diameter cannot be distinguish on CT-scans. If the $D_{\max }$ exceeds a statistically-based threshold of $55 \mathrm{~mm}$ for men and 45-50 for women 13 or if AAA $D_{\max }$ growth exceeds $1 \mathrm{~cm} \mathrm{yr}^{-117}$, patients will undergo open or endovascular aneurysm repair. Otherwise a yearly control is performed.

New guidelines ${ }^{18}$ define a more complex follow-up and repair decision process, highlighting the difficulty to predict AAA evolution based on its current diameter. $D_{\max }$ is an imperfect criterion as the estimated annual rupture rate of 4.0 to $4.9 \mathrm{~cm} \mathrm{AAA}$, is non-negligible (1.0\% per year) $)^{48}$ and $23 \%$ of ruptured AAA are less than $5 \mathrm{~cm}^{\underline{20}}$. In contrast, rupture rate in large aneurysms could be lower than expected with annual rupture rate of $3.5 \%$ for 5.5 to $6 \mathrm{~cm}$ and $4.1 \%$ in 6 to $7 \mathrm{~cm} \mathrm{AAAs}{ }^{48}$. These data show the maximal diameter/rupture relationship to be nonlinear and inaccurate to predict rupture ${ }^{28}$. Identifying better performing metrics is an active research field 34 . Risk-linked predictors are usually based on geometric shape, mechanical tissue properties and flow topology thanks to the increased availability of patient-specific 3D AAA models from computed tomography angiography (CTA). Several fields can even be combined in multi-physics and multi-scale modelling making it possible to simulate AAA growth 29167 by coupling the biology and mechanics of the disease. Known metrics of interests are AAA volume, surface, bulge height, tortuosity and local surface curvature $^{\sqrt[55 \mid 56]{56}}$ as well as mechanical stress, intrinsically relying on tissue properties, strongly heterogeneous and nonlinear ${ }^{49}$ and also patient specific $\frac{50}{50}$. From a fluid point of view, blood flow is known to play a crucial role in AAA evolution ${ }^{5415}$, as well as ILT presence and 
growth 3017 .

Very few studies focus on local parameters and their variations between two scans. Tzirakis et al. $\frac{64}{6}$ observed on six AAA, a relationship between ILT growth and Time Average Wall Shear Stress (TAWSS) and Oscillatory Shear Index (OSI) but not with Relative Residence Time (RRT), while Arzani et al. ${ }^{4}$ noticed in ten AAAs a significant relationship between ILT deposition and low OSI but not with low TAWSS. Both studies included small AAA $\left(D_{\max }<53\right.$ and $<50 \mathrm{~mm}$ respectively). Zambrano et al. ${ }^{71}$ observed a relationship between low Wall Shear Stress (WSS) and the ILT deposition locations. Furthermore on their 14 patients, ILT volume accumulation correlated with the AAA growth. The investigation of the hemodynamic mechanisms underlying AAA expansion is a promising approach to understand, and potentially provide more patient-specific tools to characterize, AAA vul-

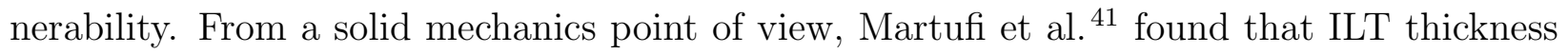
and wall stress were linked to the local growth rate.

In summary, although repair criteria alone lack patient specificity, predicting their evolution would improve clinical decision for follow-up and repair. If the disease is known to be associated with altered morphology and blood flow, intraluminal thrombus deposit and clinical symptoms, the growth mechanisms are yet to be fully understood. The goal of this work is thus to better understand AAA evolution by exploring the potential dependence between computed hemodynamics factors and morphological metrics of AAA growth on a larger longitudinal study. In this retrospective longitudinal study of 138 scans, morphological analysis and blood flow simulations for 32 patients with clinically diagnosed AAAs and several follow-up CT-scans, are performed and compared to 9 control subjects. First, the definition of a healthy group, versus low and high risk groups in terms of AAA evolution is motivated. The methods also explain the geometrical and blood flow numerical models, and define their postprocessing into global and local metrics. Global parameters distinguishing the different groups are explored, followed by local correlations between hemodynamics metrics and AAA growth. Finally, high-risk predictors trained with successively clinical, morphological, hemodynamic and all data, and their link to the AAA evolution are built 
[Figure 1 about here.]

\section{Materials and Methods}

This section first describes the patient population and associated definitions. Next, the geometrical model construction and blood flow simulation set-up are explained. Finally, postprocessing of geometry and CFD results is detailed, defining global and local metrics associated with each scan, along with the statistical analysis methods used in this study.

\subsection{Patient population and associated definitions}

This study is HIPAA (Health Insurance Portability and Accountability Act) compliant and approved by the local institutional review board (IRB) ${ }^{1}$. Since all data were anonymized, the consent form was waived by the IRB for all patients. Forty-one patients are included in the study, thirty-two with diagnosed AAA and nine healthy. Patients are considered healthy in the absence of AAA $\left(D_{\max }<30 \mathrm{~mm}\right)$ and significant arterial disease. They necessitated an abdominal scanner but without peripheral disease, were above 48 year old and were sexmatched with the AAA patients. AAA patients were selected from a clinical data base of patients having CT follow-up for AAA in our institution. The inclusion criteria for AAA patients were: $\mathbf{1}$. AAA of more than $30 \mathrm{~mm}, \mathbf{2}$. At least one baseline $\mathrm{CT}$ and 2 following $\mathrm{CT}$ scan examinations, 3. All selected CT scans were acquired with contrast injections and with a slice thickness of less than $2.5 \mathrm{~mm}$ in order to ensure accurate and efficient segmentation of the lumen and ILT.

This retrospective study does not include ruptured AAA. Usually patients with ruptured AAA are rarely followed by CT-scan as AAA is usually undiagnosed in such cases. Moreover

\footnotetext{
${ }^{1}$ Approval \#12.153 from the research ethics committee of the University of Montreal Health Centre (CHUM)
} 
the AAA size $\left(D_{\max }\right)$ can be influenced by AAA deflation following rupture and AAA outer wall is more difficult to evaluate in presence of a periaortic hematoma. $D_{\max }$ value and growth are therefore combined as a surrogate risk metric. In the text the term risk will refer to this definition and not rupture risk.

The AAA population is classified in high and low-risk populations based on the recognized criteria of AAA size and growth over time ( $D_{\max }$ and $D_{\max }$ growth). The commonly used clinical thresholds to indicate an open or endovascular surgery are a $D_{\max }$ of $55 \mathrm{~mm}$ for male and $45-50 \mathrm{~mm}$ for women and a growth of more than $5 \mathrm{~mm}$ in 6 months ${ }^{17}$. New guidelines ${ }^{18}$ temper these thresholds, indicating the need for more personalized approaches. There is less consensus on the growth threshold. Growth rate has been reported to be around $2 \mathrm{~mm} \mathrm{yr}^{-11159}$. The $5 \mathrm{~mm} /$ year growth threshold has previously been recognized as a fast growth criterion ${ }^{25}$ and this growth variation is above the $95 \%$ of the confidence interval of $D_{\max }$ measurement error $[58$. To define high risk at scan time $n$, we thus choose 'either $D_{\max }^{n}$ is over $50 \mathrm{~mm}$ for women and $55 \mathrm{~mm}$ for men, or $D_{\max }$ variation $\left(D_{\max }^{n+1}-D_{\max }^{n}\right.$ between consecutive scans) is above $5 \mathrm{~mm} \mathrm{yr}^{-1}$. A patient is considered at low risk if he/she is not at high risk. Cases are considered as high risk, as soon as one of the high risk criteria is met. For a patient at low risk, if this occurs, the patient switches to high risk for the rest of the follow-up scans.

\subsection{Geometrical model construction}

For all scans, lumens are extracted by an active contour method implemented in ITKSnap ${ }^{70}$. Aortic models include part of the suprarenal aorta including the ostia of coeliac trunk, mesenteric artery and renal arteries, as well as infrarenal aorta, and internal and external iliac arteries. ILTs are segmented using ORS Visual ${ }^{37}$, which is based on active snake segmentation. 


\subsection{Blood flow simulation set-up}

The incompressible Navier-Stokes equations are solved in each aortic model as detailed in $\underline{34}$. The flow is considered laminar, homogeneous and non-Newtonian, the viscosity following the Quemada model ${ }^{40 \mid 35}$. Model parameters are chosen according to the study of Buchanan et al. ${ }^{14}$ based on the rheological data from Kaibara et al. ${ }^{35}$. Peak Reynolds numbers of $1700-$ 2000 at the proximal inlet in the simulations are within the physiological range $\mathrm{e}^{28}$ as well as the Womersley numbers, ranging from 10 to $15^{44}$. A generic flow rate is imposed at the inlet ${ }^{43}$ (see Figure I) with Womersley profile ${ }^{66}$. The lumen is defined as the space inside the aorta, either bounded by the ILT or the arterial wall. Both are considered rigid and a no-slip condition for the blood is imposed on the boundary they form. The ILT is thus excluded from the computational domain. Complex re-circulation patterns oftentimes exists stretching up to the outlet planes. Additionally, reverse flow during diastole ${ }^{27}$ is likely to create numerical instability. Gradient stabilization to control complex backflow in the domain similarly to Bertoglio et al. $[$ is implemented. At outlets, an RCR Windkessel model is applied (see Table I in Supplementary materials for parameter value). The domain is discretized using a polyhedral mesh with refined boundary layers around 0.8-1 million elements (edge length $\approx 0.35 \mathrm{~mm}$ ), and the Navier-Stokes equations are discretized with finite volume methods (FVM) implemented in the OpenFOAM toolbox. The convective and diffusive term are discretized using a second order Gauss scheme and the time scheme is Crank-Nicholson, also second order ${ }^{31 / 32}$. The solver is a large time-step transient solver for an incompressible fluid for solving pressurevelocity coupling, the PIMPLE (merged PISO-SIMPLE) algorithm. The solution is considered converged if:

- each time step is fully converged under chosen residuals criteria, i.e. $10^{-6}$ for pressure and $10^{-8}$ for velocity; an adaptive time-step was used with the $C F L<1$ criteria.

- the periodic convergence is achieved, typically after 5-7 cardiac cycles.

- the solution (velocity, WSS) is independent of further mesh refinement, computed using the Grid Convergence Index (GCI) 


\subsection{Definition of global and local metrics}

Clinical metrics. The clinical metrics are listed in Table 1. Their availability among the patient population is reported in the same table.

Lumen centerline and patch description. Most morphological metrics rely on the computation of the centerline of the lumen. The centerline is extracted with VMTK ${ }^{2}$, which is based on the Voronoï diagram decomposition of the lumen. The subdomain of interest, i.e. the lumen between the lower renal artery and the iliac bifurcation, is automatically extracted by splitting the surface using the centerline bifurcation information ${ }^{1}$. It allows a reproducible domain split necessary for surface and volume comparison. Once extracted, the lumen is split along its rotational (24 divisions or $15^{\circ}$ ) and longitudinal axis, with respect to the centerline curvature (25 divisions), resulting in 600 patches $^{3}$ (see Figure 2 for the method and Figure II in Electronic Supplementary Material for an example). All fields defined on the lumen are averaged on each subdivision. Assuming spatial deformation is spatially homogeneous between acquisitions, each averaged field is compared to its value at the next time step at the estimated same location. Local change is thus computed on a grid-like array : it is the patch-wise variation.

The statistical analysis is thus divided in the following manner:

- Unique value for each AAA, (e.g. ILT volume) and its annual variation ; see Table 2 and Table 3

- Spatially distributed metrics, such as TAWSS. First, the distribution information is reported (extrema, average and standard deviations, see Table 4), and then the patchwise annual variation, also reported as extrema, average and standard deviation. For example, the local change of $O S I_{\max }$ refers the maximal change of OSI value from one patch at time $t$ to the same patch at time $t+1$. See Table 5 and Table 6 .

[Figure 2 about here.] 
Morphological metrics. To characterize the AAA morphology, we consider (see Table II for definition and references): 1) the maximal lumen diameter $D_{\text {max }}^{\text {lumen }}$, computed as the maximal diameter of the AAA luminal sections, defined perpendicular to the lumen centerline, 2) the maximal diameter $D_{\max }$ measured at the outer wall, computed normal to the outer wall centerline, 3) the ILT thickness (local and global metric), computed as the Euclidean distance between the lumen and the outer AAA wall, 4) the lumen centerline curvature, 5) the lumen centerline tortuosity, 6) the Normalized Shape Index (NSI) which characterizes the deviation from a sphere (NSI =1). As the arterial wall is not discernible from the ILT, it is considered of constant thickness and subtracted from the measured distance. The wall thickness covered by ILT is chosen of a constant value of $1 \mathrm{~mm}^{33}$. A few other metrics are also considered. The lumen surface and volume are defined by the surface area and the volume of the portion of the aortic lumen comprised between the lowest renal and the iliac bifurcation. The ILT volume is also computed for the same region of interest, and the total aortic volume is the sum of lumen and ILT volumes. The ILT coverage is defined as the percentage of the lumen covered with ILT, computed from the number of surface patches on which the average ILT thickness was over one millimetre. The local growth criteria, as defined in the patch-description paragraph, is the ILT thickness change ${ }^{60}$.

Hemodynamics metrics. Finally, to evaluate the flow alteration at the wall, the TAWSS, OSI, RRT and Endothelial Cell Activation Potential (ECAP) are computed from the WSS at the lumen wall (see Table II), leading to local and global metrics as defined in the patchdescription paragraph.

\subsection{Statistical analysis}

Global descriptive statistics. First, descriptive statistics are performed to report population characteristics with a univariate analysis to compare patient populations Figure 1. The population is divided into three groups, as defined in section 2.1: control cases without AAAs, cases with AAA but considered at low-risk, and cases with AAA at high-risk. Potential 
correlation between groups for each variable is computed by a Welch's t-test. Similar to the Student's t-test, it accounts for the unequal variance between the samples. Samples are normally distributed as required by the test. In the results, the tables report the lists of variables or global metrics used to describe the AAA and the distribution of their values among the healthy $(\mathrm{H})$, low-risk (LR) and high-risk (HR) patients. For each variable, a Welch's t-test is performed between the healthy and low-risk groups (H-LR), the healthy and low-risk groups (H-HR) and the low-risk and high-risk groups (LR-HR). When significant difference is observed $(p<0.05)$ between two groups, it is reported in the $4^{\text {th }}$ column. Annual variation of hemodynamic parameters and thrombus thickness are computed locally; i.e. patch to patch.

Local descriptive statistics Descriptive statistics are also performed locally (patch-wise) to evaluate the relationships between flow and local morphological metrics Figure 1, in terms of local Euclidean lumen border distance to the centerline (thereafter called 'distance to the centerline'), ILT thickness and patch surface area. Unsupervised outlier detection is performed on each dataset with the Local Outlier Factor (LOF) method ${ }^{12}$. As described in Rowland et al. in particular for WSS ${ }^{51}$, local correlation between a phenomena and a bio-mechanical metric is hindered by spatial auto-correlation. One reported alternative is bootstrapping ${ }^{26}$ and performing the statistical test on the new dataset. Here, repeating 10000 times the non-parametric Spearman test yields reproducible results. Considering the large amount of data, distribution of Spearman's $\rho$ is reported. Indeed, with a large enough sample size, a very weak correlation can be significant, when the observed effect is likely not real and due to chance in a statistical sense.

Risk predictor methods. Next, we try to anticipate the behaviour of AAA, i.e. to predict the risk based on current information Figure 1. The (predicted) risk criteria is therefore chosen to account for the state after the time evaluated $t^{n}$ : the $D_{\max }$ variation was conserved $\left(\left(D_{\max }^{n+1}-D_{\max }^{n}\right) /\left(t^{n+1}-t^{n}\right)\right)$ but the $D_{\max }$ considered is $D_{\max }^{n+1}$. The thresholds defining low versus high risks are the same as in section 2.1. 
To better understand the contribution of the different groups of metrics on risk assessment, the predictor is first built using each set separately, i.e. $D_{\max }$ only, clinical, morphological and hemodynamic metrics and then all mixed. The features (input layer) are combined using a neural network to classify whether the next time step is at high-risk or not (output layer). Back-propagation is used to determine weights. Here, a multi-layer perceptron network, implemented in Theano ${ }^{63}$ is used. The ten features explaining the most the dataset variance are chosen based on a Principal Components Analysis (PCA). This prevents having too many features compared to the number of samples and the associated risk of overfitting. Hyperparameters are automatically tuned ${ }^{61}$ to maximize the Area Under the Curve (AUC) of the Receiver Operating Characteristic (ROC) curve and f1-score.

To evaluate the five estimators performance and avoid overfitting, we run repeated kfold cross correlation with $k=3$ and 10 repetitions. Features finally selected for each set are reported in Figure 8. For clinical interpretation of the results, the ROC curves with AUC value, and the relative rank of features with respect to the predictability of the target variable evaluated by a multi-class $A d a B o o s t^{15}$, are reported. AUCs medians are compared with the Delong et al. ${ }^{21}$ method.

\section{Results}

\subsection{Population description}

Healthy population mean age is estimated at $60.4 \pm 12.4$ years while AAA patient mean age is $73.5 \pm 7.4(p<0.05)$. Among the AAA patients, 5 are women $(15.6 \%$, mean age $73.6 \pm 9.4$ years $)$ and 27 men $(84.4 \%$, mean age $73.5 \pm 7.0$ years, $(p=0.98))$ while in the healthy group, one is a woman $(11.2 \%, 78$ years) and 8 are men ( $88.8 \%$, mean age $58.4 \pm 11.6$ years). At least three follow-up CT-scans are available and suitable for domain reconstruction for AAA patients (mean $4 \pm 1.47$, range 3-9). Mean time between follow-up CT is $12.74 \pm 12.41$ months (range : 0.16 to 79.63 months and one case where two CTs were performed on the same day) and the mean follow-up duration is $38.62 \pm 22.53$ months (range : 6.35 to 111.42 
months). Clinical data is available for most patients (see Table 1).

[Table 1 about here.]

Regarding the healthy group, $D_{\max }$ is $18.2 \pm 3.71 \mathrm{~mm}$ (range: $14.5-27.45 \mathrm{~mm}$ ) whereas in AAA patients $D_{\max }$ is estimated $42.68 \pm 8.39 \mathrm{~mm}$ (range: $22.65-67.49 \mathrm{~mm}$ ). At baseline, $7(21.9 \%)$ AAA $D_{\max }$ are over the high-risk threshold and $4(12.5 \%)$ have a growth over $5 \mathrm{~mm} /$ year ; $2(6.25 \%)$ achieve both. At the last exam, and 16 (50\%) are over the high-risk $D_{\max }$ threshold and $9(28.1 \%)$ have reached the high-risk growth threshold at the previous follow-up (defined as $\left.D_{\max }^{n+1}-D_{\max }^{n}\right) ; 7(21.9 \%)$ achieve both. The lumen of the healthy aortas are shown in Figure 3. Most of them present various degrees of tortuosity, their length increasing with age ${ }^{69}$.

\subsection{Distribution of clinical, morphological and hemodynamic pa- rameters in the studied population}

[Figure 3 about here.]

This section first studies how metrics associated with the different scans vary among the population and which ones distinguish the different patient groups (see Figure 1). The distribution of the clinical, morphological and hemodynamic variables among the three groups is presented in tables ( Table 2, Table 3, Table 4, Table 5 and Table 6 ) as well as the correlations between groups for each variable.

139 CT-scans and simulations, from 41 patients, are split into three study groups (healthy $(\mathrm{H})$, low (LR) and high-risk (HR)) as defined in the Methods section. The LR and HR groups include 59 and 70 cases respectively. Univariate analysis reveals that the $D_{\max }$ as well as 10 other variables significantly separate all three groups, some of those being highly correlated, such as volumes and diameters. 18 variables could significantly separate the healthy from the low-risk group, 18 the healthy from the high-risk group, and 27 the low-risk from the high-risk group. For the clinical metrics, all but BMI, separate the groups: pressure for low vs high 
risks, age, dyslipidemia and statins for healthy vs the other groups. Regarding morphology, all metrics defined for healthy and AAAs can separate the three groups, except for the lumen shape factor (NSI) which separates healthy from AAA but not low-risk from high-risk AAA. Among AAAs, ILT metrics separate low from high risk groups, except ILT coverage and minimum ILT thickness. Regarding annual variations, $D_{\max }$ as expected distinguishes the two groups but among all other metrics, only local change of minimum and maximum ILT thickness make that difference, hinting on a particular role of ILT in the local growth process that we will explore in the next section. For all these morphological metrics, significant difference is achieved mostly from mean values but not from their standard deviation (all but the $\left.D_{\max }\right)$. In contrast, for the fluid-based metrics, Table 4 and Table 6 , both average values and standard deviations can discern groups (e.g. the $E C A P_{\max }$ and local $E C A P_{\max }$ variation). Almost all hemodynamics variables have several metrics that separate healthy from AAAs. TAWSSmin, RRTmean, ECAPmax,mean,stdev further separate low from highrisk groups. Regarding local changes, minimum metrics always separate the two groups, as well as RRTmax and ECAPmax. These results suggest that both instantaneous metric values and their changes are important to understand growth.

[Figure 4 about here.]

In Figure 4, the segmented lumen and ILT of two patients during their follow-up are shown. The one on the left shows no major shape change or growth with time, with a fusiform shape and a thin ILT. For the right one, ILT becomes more saccular with time and lumen thinner. This major difference in behaviour is plotted in Figure 5 for all patients during their follow-up. $D_{\max }, D_{\max }^{\text {lumen }}$, lumen tortuosity and shape index plots illustrate the important diversity of the population. The difference between groups is detailed in Table 3 and Table 5 .

[Table 2 about here.]

[Table 3 about here.] 
[Table 4 about here.]

[Table 5 about here.]

[Table 6 about here.]

[Figure 5 about here.]

[Figure 6 about here.]

[Figure 7 about here.]

Next, we present results to understand if there is a local correlation between morphological and hemodynamic metrics that could explain local growth Figure 1. Figure 7 describes the correlation coefficients between the wall metrics and two morphological metrics, computed on all patches of each scan : the distance from the lumen wall to the centerline, normalized by the proximal i.e. near the renal arteries distance, and the ILT thickness. There is a large dispersion between patients and scans (see Figure III for the individual data). As a consequence, some exhibit nice correlations (Figure 6a), while others do not (Figure 6r). The large dispersion of Spearman's $\rho$ distribution is illustrated in Figure 6 for one metric. Despite a very large dispersion of data for both the distance to the centerline and the ILT thickness, visible trends stand out (Figure 7). TAWSS and WSSG strongly negatively and OSI positively correlate with the centerline distance. Coherently, RRT and ECAP also present strong positive correlation with the distance to the centerline. RRT and TAWSS distributions do not include $\rho=0$. Regarding ILT thickness, no strong correlation emerges. TAWSS negatively correlates with ILT thickness. This finding is coherent with the common knowledge of low WSS being linked to thrombogenesis. WSSG and OSI also show slightly negative correlation with ILT thickness while no conclusion can be drawn from the RRT and ECAP $\rho$ distributions. In fact, all five hemodynamic metrics $\rho$ distributions are divided between positive and negative values, sign of a great heterogeneity between scans. When the statistics are computed on each patient instead of each scan separately, trends are conserved with however lower dispersions (Figure 7, bottom). 


\subsection{Global classification as a risk predictor}

Finally, we study if the AAA evolution can be predicted (Figure 1). The ability of the classier to discern future high-risks from low-risks cases is presented in Table 2. Recall that this prediction is based on features of the current time. The features are initially divided into 5 sets: $D_{\max }$ only, clinical, morphological and hemodynamic separately and then all features merged. The relative influence of the individual features on the dataset is also plotted for a better understanding of their role.

For reference, the classification is evaluated with $D_{\max }$ only and the corresponding AUC is $0.75 \pm 0.08$. No feature ranking is present as the entire classification information comes from $D_{\max }$. For clinical features alone, the AUC is $0.73 \pm 0.09$ and the most separating features are age, $p_{\text {sys }}$ and $p_{\text {dias }}$. With only morphological features ( $D_{\text {max }}$ excluded), the AUC is $0.93 \pm 0.09$. The information mostly comes from the lumen centerline curvature, the ILT volume and thickness, and the lumen NSI. Concerning hemodynamic features, the AUC is $0.96 \pm 0.10$ with information mostly gained from $O S I_{\text {mean }}, E C A P_{\text {stdev }}, R R T_{\max }$ and $E C A P_{\max }$. Finally, with all features combined, the AUC reaches $0.98 \pm 0.06$ : information is mostly gained from ILT volume, $O S I_{\max }, O S I_{\text {mean }}$ and $W S S G_{\text {stdev }}$. To evaluate the statistical difference between features sets, p-values between AUCs are computed and reported in Table 7. Significant differences are observed when all the features are compared to a single feature class, and also when the flow features are compared to the $D_{\max }$.

[Figure 8 about here.]

[Table 7 about here.]

\section{Discussion}

Quantifying AAA rupture risk has been an active field of research for at least the last decade without dethroning the $D_{\max }$ criteria. However, parameters accompanying the disease progression have been observed and discussed, including clinical observation, morphology, struc- 
tural or fluid analysis and a mix of those. High risk AAA are essentially either undiagnosed or repaired, hence the scarcity of longitudinal studies with very fast growing aneurysms. If metrics such as WSS or arterial wall solid stress alone cannot specifically single out high-risk aneurysms, their influence on the temporal evolution of AAA may be more relevant ${ }^{58}$.

In this work we have attempted to find potential underlying relationships between clinically available variables or computed metrics that quantitatively characterize AAA and their hazardous growth. An AAA is considered at risk after reaching a threshold $D_{\max }$ or exceeded a $D_{\max }$ monthly variation threshold. If such relationship exists, one can envision a new combination of parameters to be a reliable predictor of an AAA evolution from a single scan, and thereby enhancing the patient-specific decision-making process about increased surveillance or type of treatment.

\subsection{Descriptive statistics}

A total of 129 AAA from 32 patients and 9 healthy aortas were considered. The nonnewtonian flow was simulated with FVM including backflow stabilization, and all WSS-based fields and geometrical metrics were discretized on the patch-parameterized AAA lumen. All data were mapped onto the same patch space to be able to compute local time variation of metrics. When exploring the local relationships, i.e. patch-wise, both WSSG and TAWSS negatively strongly and OSI positively correlate with the local distance from the lumen wall to the centerline. This distance is normalized for each scan by the distance at the proximal neck of the AAA. This local distance thus contains information on the local dilation of the AAA, likely creating low TAWSS and high OSI zones. RRT and ECAP, by construction, have an opposite behavior from the OSI and TAWSS. By contrast, it is difficult to conclude for the local relationships with the ILT thickness given the variation among scans. Correlations with the annual variation of the lumen wall distance to the centerline and ILT thickness were not reported, presenting no visible trend. Considering all local variables may not be the appropriate measure to understand local growth. However, the risk prediction based on hemodynamic features works well (see section 3.3): mean, extrema or standard deviation of 
the local metrics seem to be the overall drivers for AAA growth. Nonetheless, previous work, especially Tzirakis et al. ${ }^{64}$ found weak relationship between ILT growth and TAWSS (see Figure 7). However, data from their study do not reveal correlation with low OSI as weakly shown in Figure 7 and Arzani et al. ${ }^{4}$ did. TAWSS seemed relevant in both studies as well as in Zambrano et al. ${ }^{71}$. These studies considered few patients. To our understanding and as Figure 7 illustrates, correlations with local morphological growth are highly heterogeneous among patients, even if for a given patient strong relations can emerge. This behavior prevents emanation of general correlations. Finding a relevant normalization space between all patients may lead to a better understanding of the local growth causes.

Figure 5 and Figure III illustrate the variety of situations encountered by clinicians when following an AAA over a given period of time. When all patient data is overlaid, no group separation visibly arises from the curves. However, from Table 2 to Table 6 one can observe that many parameters can separate patients; especially 27 of them can discern the high from the low-risk group. As expected, the classical $D_{\max }$ was one of the parameters sensitive enough to discern healthy aortas from low-risk AAAs, and also low from high-risk AAAs. ILT and total volume present the same capacity, as all three are directly linked. Despite the tendency of ILT to fill the AAA cavity, tending to reshape the lumen into a more tubular fashion, Figure 5 shows that lumens of AAA at higher risk tend to be more tortuous with a larger $D_{\max }^{\text {lumen }}$. At the same time, while for low-risk AAA the $D_{\max }^{\text {lumen }}$ shows little to no growth, the $D_{\max }$ is continuously increasing. A major hypothesis to explain AAA growth is acceleration of the loss of mechanical properties of the aortic wall due to ILT deposition. ILT leads to local wall hypoxia and inflammation ${ }^{62}$, smooth muscle cells apoptosis, elastin degradation and MMP-2 (matrix metalloproteinase-2) concentration. Shifting the pressure load normally mostly borne by elastin cells to collagen fibers contributes to the wall stretching and diameter increase. The shape modification can lead to an increase of the lumen surface prone to ILT deposition, thus maintaining the vicious cycle. Figure 5 could indicate that some AAA could remain at low-risk provided that their lumen keeps its shape and size relatively constant, and the $D_{\max }$ growth remains below the repair threshold. This could 
lead to a better understanding of the difficulty to assess AAA risk, given the presence of patients with large AAAs who will have a lower proportion of rupture than expected $\frac{48}{4}$ and relatively small aneurysms that rupture ${ }^{20}$.

Currently, sex-adjusted $D_{\max }$, absolute value and progression, is obviously significantly associated with the high risk population. Surprisingly volume and surface progression, despite a theoretically higher sensitivity, were not associated with patient risk. Similarly, no other morphological metrics annual variation could, despite higher theoretical sensitivity such as volume and surface versus diameters. One hypothesis is that the cumulative segmentation error induces a higher variability than the observed growth, especially for slow growing AAAs.

Low TAWSS and high OSI are linked to atheroprone regions of AAAs and predominate at site of rupture ${ }^{10}$. Di Achille et al. ${ }^{24}$ combined both to form the ECAP. This metric does not offer a mechanistic explanation on ILT deposition but more an imprint on the wall of the near wall flow features that are related to thrombus deposition. Additionally, AAA wall is mostly covered by ILT, and if not, is highly atherosclerotic; therefore seeking metrics related to the wall mechano-adaptation resulting from endothelial cell triggering may not be successful. However, low wall shear can inform about two different phenomena : it is the imprint on the wall of the local flow alteration, and it also favors activated platelets adhesion. As expected, $E C A P_{\max }$ and $E C A P_{\text {mean }}$ can separate the population but, interestingly, also $E C A P_{\text {stdev }}$. Standard deviations were added for all metrics, motivated by the highly patient specific data distribution (see Figure III to Figure VI) and can be considered as an indicator of the wall roughness. CT-scan resolution cannot report wall roughness due to atherosclerosis and smoothing prior to meshing removes any small scale perturbation. Nonetheless, larger scale perturbation persists that cannot be explained. Hypotheses include poor segmentation of calcifications, often overestimated on CT-scans, contrast inhomogeneity, recurrent in large blood filled cavities such as AAAs or real morphological alteration. Numerically the WSS is computed using the vector normal to the wall, at each cell, and is therefore highly dependent on surface quality. However, because WSS derived metrics allow to statistically separate 
groups and being visually consistent (e.g. see the ECAP distribution plot in Figure VI), we believe the geometrical perturbation is likely of biological origin.

Looking at the local change, i.e. patch-wise, is more challenging. Significant variations of $O S I_{\min }, T A W S S_{\min }, W S S G_{\min }, R R T_{\min }$ and $E C A P_{\min }$ are negative and average values are lower for high-risk than for low-risk patients. Whereas for ECAP, mean values increase faster for high-risk AAA while $E C A P_{\max }$ is increasing faster but $E C A P_{\min }$ is decreasing faster too. This indicates a larger dispersion of the values with time for high-risk AAA than for low-risk AAA, explaining the added value of the standard deviation of variables.

\subsection{Risk prediction}

The classification process aimed at building a risk predictor based on information acquired at a given time to anticipate if the patient will evolve to a high-risk state or stay at low-risk in the foreseeable future. Knowing that many of the evaluated metrics of interest contain powerful information to separate the low from the high-risk population, but does not give better results than the $D_{\max }$ if taken alone, a combination of metrics was sought.

For reference, we started with the $D_{\max }$ alone as feature to predict the future risk, providing a mediocre yet above the average predictor. Clinical information did not perform well either but the missing clinical features for some patient may have a large impact on the predictor and results shall be taken cautiously. However, age, systolic and diastolic pressure and BMI are known factors associated with AAA risk. When the morphological and hemodynamic features are considered separately, the predictor performs well, even with the repetition of the 3 -folds splits on a small cohort of patients. Once all features are merged, the AUC reaches 0.95. However, despite high values of AUCs for flow and morphological features, Table 7 shows that taking either flow metrics or all features leads to a significant difference with using the $D_{\max }$ alone. We believe that the very conservative results of the p-values (in Table 7) comes from the variability of the AUCs during the k-fold repetition (visible in Figure 8). A larger patient database with a prospective follow-up should confirm the clinical relevance of the AUC obtained here. However, morphological features can now 
be easily obtained by lumen and thrombus segmentation ${ }^{37}$ for a significant increase in classification power. Even if the relation between flow alteration and AAA growth is still poorly understood, the combination of flow pattern with morphological analyses clearly improves patient risk stratification and should be integrated in future clinical algorithms.

\subsection{Limitation}

This study presents limitations discussed below:

- Simulation did not include wall deformation due to the pressure variation during the cardiac cycle. While FSI models for the aorta exist, the aortic wall was considered rigid, in accordance both with previous measurements ${ }^{34}$ and literature ${ }^{49}$. Also, in the context of diseased aortas, the wall is highly heterogeneous and no non-invasive measurement can currently capture such heterogeneous mechanical properties.

- Boundary conditions are literature-based as this was a retrospective study. Thus, no patient-specific measurement was available, as often in such cases. However, flow patterns were favorably compared with PC-MRI data on a few patients with $\mathrm{AAA}^{34}$.

- For some patients the $D_{\max }$ profiles are not monotonically increasing. However, it seems to be unlikely that small diameter reduction $\left(\Delta D_{\max }<2 \mathrm{~mm}\right)$ is in fact outer wall shrinkage. Several possible explanations include: segmentation fluctuation localized on artifacts or calcifications; AAA shape change due to the patient positioning in the scanner, altering the observed geometry, thus the maximal diameter; the limitation of the measurement approach using planes normal to the centerlines in complex shaped AAAs. Considering the inter-observer agreement of $<3 \mathrm{~mm}$ on the $D_{\max } \frac{37}{\text {, the same }}$ error tolerance is expected by using the same segmentation tool.

- While flow in healthy aortas remains essentially laminar, complex flow $\underline{46}$ and transition to turbulence ${ }^{\sqrt{3865}}$ may occur in AAAs due to the brutal enlargement and could impact the studied wall fields. 
- To characterize AAA growth, metrics were compared patch to patch which does not reflect the non-homogeneous and anisotropic growth of AAA. In the absence of local wall displacement tracking method, this approach still gives insights on AAA growth.

- Considering the number of follow-up scans available, the learning approach did not considered AAAs as time-series. An approach similar to Lipton et al. ${ }^{39}$ could eventually be implemented on a database with more follow-up scans per patient.

- The healthy population was 13 years younger than the AAA population on average (but with aortas already showing signs of aging, see Figure 3) and clinical data was not available for all patients, see Table 1 .

- The database did not include ruptured aneurysms for the reasons described in the Methods section. When such data become available for AAA, the link between 'high risk' as defined here by clinicians and rupture prediction should be studied. A very recent study in cerebral aneurysms showed adverse morphology and hemodynamics to be related to aneurysm rupture ${ }^{22}$. The corresponding statistical model of rupture probability was then successfully validated ${ }^{23}$. These results combined with our findings give hope that such approach should be successful for AAAs as well.

\subsection{Conclusion}

We have presented a retrospective population study on the metrics quantifying the growth of AAA, and have built a model to anticipate their further evolution towards rupture. This longitudinal study included clinical and imaging data available at different time points for a total number of 138 scans from 42 patients. The analysis considered clinical, morphological and simulation-based hemodynamic metrics, separately or combined to incorporate a diversity of potential growth markers. Different global and local metrics or their time evolution were found to separate the healthy, low-risk and high-risk groups. Local hemodynamics metrics presented in fact a large intra- and interpatient variability: even if for some patients a clear relationship could be established between hemodynamics variables 
and growth, their extrapolation to the whole population is yet to be found. Nevertheless, a risk predictor could be built with supervised learning from the clinical, morphological and simulation-based hemodynamic metrics. From a clinical point of view, we have shown that, compared to the current clinical criteria, morphological metrics describing the lumen and ILT shape could already greatly improve risk prediction, and thus potentially patient follow-up or treatment decision, at a moderate analysis cost. Blood flow simulations provide valuable additional information for the predictor, as well as for understanding the underlying relationship between flow alteration and AAA growth. Finally, risk prediction works best by combining all metrics. Although the results show the high predictive value of this approach, validation of the risk predictors on another set of data is needed before clinical translation.

\section{Acknowledgments}

The authors thank Daniel Stubbs and Compute Canada for the HPC support. The presented work relies on open-source projects and their community, especially OpenFOAM and ITKSnap (http://www.itksnap.org). This work has been supported by the Collaborative Research and Development Grants no. 460903-13 provided by the Natural Sciences and Engineering Research Council of Canada (NSERC) and the Industry-partnered Collaborative Research grant no. 124294 from the Canadian Institutes of Health Research (CIHR).

\section{References}

1. Antiga, L., B. Ene-Iordache, and A. Remuzzi. Computational geometry for patientspecific reconstruction and meshing of blood vessels from MR and CT angiography. IEEE Transactions on Medical Imaging 22:674-684, 2003.

2. Antiga, L. Patient-specific modeling of geometry and blood flow in large arteries. Politecnico di Milano, 2002. 
3. Antiga, L. and D. A. Steinman. Robust and objective decomposition and mapping of bifurcating vessels. IEEE Transactions on Medical Imaging 23:704-713, 2004.

4. Arzani, A., G.-Y. Suh, R. L. Dalman, and S. C. Shadden. A longitudinal comparison of hemodynamics and intraluminal thrombus deposition in abdominal aortic aneurysms. American Journal of Physiology-Heart and Circulatory Physiology 307:H1786-H1795, 2014.

5. Basciano, C., C. Kleinstreuer, S. Hyun, and E. A. Finol. A relation between near-wall particle-hemodynamics and onset of thrombus formation in abdominal aortic aneurysms. Annals of Biomedical Engineering 39:2010-2026, 2011.

6. Bertoglio, C., A. Caiazzo, Y. Bazilevs, M. Braack, M. Esmaily, V. Gravemeier, A. L. Marsden, O. Pironneau, I. E. Vignon-Clementel, and W. A. Wall. Benchmark problems for numerical treatment of backflow at open boundaries. International Journal for Numerical Methods in Biomedical Engineering , 2018.

7. Bhagavan, D., P. D. Achille, and J. D. Humphrey. Strongly Coupled Morphological Features of Aortic Aneurysms Drive Intraluminal Thrombus. Scientific Reports pp. 1-18, 2018.

8. Björck, M. and A. Wanhainen. Pathophysiology of AAA: Heredity vs Environment. Progress in Cardiovascular Diseases 56:2-6, 2013.

9. Bluestein, D., L. Niu, R. T. Schoephoerster, and M. K. Dewanjee. Steady Flow in an Aneurysm Model: Correlation Between Fluid Dynamics and Blood Platelet Deposition. Journal of Biomechanical Engineering 118:280, 1996.

10. Boyd, A. J., D. C. Kuhn, R. J. Lozowy, and G. P. Kulbisky. Low wall shear stress predominates at sites of abdominal aortic aneurysm rupture. Journal of Vascular Surgery 63:1613-1619, 2016. 
11. Brady, A. R., Thompson, S. G., Fowkes, F. G. R., Greenhalgh, R. M., and Powell, J. T. Abdominal aortic aneurysm expansion: risk factors and time intervals for surveillance. Circulation, 110(1), 16-21, 2004.

12. Breunig, M. M., H.-P. Kriegel, R. T. Ng, and J. Sander. LOF: Identifying DensityBased Local Outliers. Proceedings of the 2000 Acm Sigmod International Conference on Management of Data , 2000.

13. Brewster, D. C., J. L. Cronenwett, J. W. Hallett, K. W. Johnston, W. C. Krupski, and J. S. Matsumura. Guidelines for the treatment of abdominal aortic aneurysms: Report of a subcommittee of the Joint Council of the American Association for Vascular Surgery and Society for Vascular Surgery. Journal of Vascular Surgery 37:1106-1117, 2003.

14. Buchanan, J. R., C. Kleinstreuer, and J. K. Comer. Rheological effects on pulsatile hemodynamics in a stenosed tube. Computers and Fluids 29:695-724, 2000.

15. Busa-Fekete, R., B. Kégl, G. Szarvas, O. Chapelle, Y. Chang, and T.-Y. Liu. Ranking by calibrated AdaBoost. In: Learning to Rank Challenge, volume 14, pp. 37-48. 2011.

16. Chaikof, E. L., J. D. Blankensteijn, P. L. Harris, G. H. White, C. K. Zarins, V. M. Bernhard, J. S. Matsumura, J. May, F. J. Veith, M. F. Fillinger, R. B. Rutherford, and K. Craig Kent. Reporting standards for endovascular aortic aneurysm repair. Journal of Vascular Surgery , 2002.

17. Chaikof, E. L., D. C. Brewster, R. L. Dalman, M. S. Makaroun, K. A. Illig, G. A. Sicard, C. H. Timaran, G. R. Upchurch, and F. J. Veith. The care of patients with an abdominal aortic aneurysm: The Society for Vascular Surgery practice guidelines. Journal of Vascular Surgery 50:49, 2009.

18. Chaikof, E. L., R. L. Dalman, M. K. Eskandari, B. M. Jackson, W. A. Lee, M. A. Mansour, T. M. Mastracci, M. Mell, M. H. Murad, L. L. Nguyen, G. S. Oderich, M. S. Patel, M. L. Schermerhorn, and B. W. Starnes. The Society for Vascular Surgery practice 
guidelines on the care of patients with an abdominal aortic aneurysm. Journal of Vascular Surgery, 2018.

19. Chisci, E., N. Alamanni, F. Iacoponi, S. Michelagnoli, T. Procacci, G. Colombo, and C. Setacci. Grading abdominal aortic aneurysm rupture risk. The Journal of cardiovascular surgery 59:87-94, 2018.

20. Darling, R. C., C. R. Messina, D. C. Brewster, and L. W. Ottinger. Autopsy study of unoperated abdominal aortic aneurysms. The case for early resection. Circulation $56: 161-4,1977$.

21. DeLong, E. R., D. M. DeLong, and D. L. Clarke-Pearson. Comparing the areas under two or more correlated receiver operating characteristic curves: a nonparametric approach. Biometrics 44:837-45, 1988.

22. Detmer, F. J., Chung, B. J., Jimenez, C., Hamzei-Sichani, F., Kallmes, D., Putman, C., and Cebral, J. R.. Associations of hemodynamics, morphology, and patient characteristics with aneurysm rupture stratified by aneurysm location. Neuroradiology, 61(3), 275-284, 2019.

23. Detmer, F. J., Fajardo-Jimnez, D., Mut, F., Juchler, N., Hirsch, S., Pereira, V. M., and Cebral, J. R.. External validation of cerebral aneurysm rupture probability model with data from two patient cohorts. Acta neurochirurgica. Acta neurochirurgica, 160(12), 2425-2434., 2018.

24. Di Achille, P., G. Tellides, C. A. Figueroa, and J. D. Humphrey. A haemodynamic predictor of intraluminal thrombus formation in abdominal aortic aneurysms. Proceedings of the Royal Society A: Mathematical, Physical and Engineering Sciences 470:2014016320140163, 2014.

25. Duellman, T., C. L. Warren, J. Matsumura, and J. Yang. Analysis of multiple genetic polymorphisms in aggressive-growing and slow-growing abdominal aortic aneurysms. Journal of Vascular Surgery 60:613-621, 2014. 
26. Efron, B. and R. Tibshirani. Bootstrap Methods for Standard Errors, Confidence Intervals, and Other Measures of Statistical Accuracy. Statistical Science 1:54-75, 1986.

27. Esmaily Moghadam, M., Y. Bazilevs, T. Y. Hsia, I. E. Vignon-Clementel, and A. L. Marsden. A comparison of outlet boundary treatments for prevention of backflow divergence with relevance to blood flow simulations. Computational Mechanics 48:277-291, 2011.

28. Finol, E. A. and C. H. Amon. Blood Flow in Abdominal Aortic Aneurysms: Pulsatile Flow Hemodynamics. Journal of Biomechanical Engineering 123:474, 2001.

29. Grytsan, A., P. N. Watton, and G. A. Holzapfel. A Thick-Walled FluidSolid-Growth Model of Abdominal Aortic Aneurysm Evolution: Application to a Patient-Specific Geometry. Journal of Biomechanical Engineering 137:031008, 2015.

30. Haller, S. J., J. D. Crawford, K. M. Courchaine, C. J. Bohannan, G. J. Landry, G. L. Moneta, A. F. Azarbal, and S. Rugonyi. Intraluminal thrombus is associated with early rupture of abdominal aortic aneurysm. Journal of Vascular Surgery 67:1051-1058, 2018.

31. Jasak, H. Error Analysis and Estimation for the Finite Volume Method with Applications to Fluid Flows. Ph.D. thesis, Imperial College, University of London, 1996.

32. Jasak, H. and H. G. Weller. Application of the finite volume method and unstructured meshes to linear elasticity. International Journal for Numerical Methods in Engineering 48:267-287, 2000.

33. Kazi, M., Thyberg, J., Religa, P., Roy, J., Eriksson, P., Hedin, U., and Swedenborg, J. Influence of intraluminal thrombus on structural and cellular composition of abdominal aortic aneurysm wall . Journal of vascular surgery, 38(6), 1283-1292, 2003.

34. Joly, F., G. Soulez, D. Garcia, S. Lessard, and C. Kauffmann. Flow stagnation volume and abdominal aortic aneurysm growth: Insights from patient-specific computational flow 
dynamics of Lagrangian-coherent structures. Computers in Biology and Medicine 92:98$109,2018$.

35. Kaibara, M. Rheology of blood coagulation. Biorheology 33:101-117, 1996.

36. Himburg, H. A., D. M. Grzybowski, A. L. Hazel, J. A. LaMack, X. M. Li, and M. H. Friedman. Spatial comparison between wall shear stress measures and porcine arterial endothelial permeability. American Journal of Physiology-Heart and Circulatory Physiology, 286(5), H1916-H1922, 2004.

37. Kauffmann, C., A. Tang, A. Dugas, E. Therasse, V. Oliva, and G. Soulez. Clinical validation of a software for quantitative follow-up of abdominal aortic aneurysm maximal diameter and growth by CT angiography. European Journal of Radiology 77:502-508, 2011.

38. Les, A. S., S. C. Shadden, C. A. Figueroa, J. M. Park, M. M. Tedesco, R. J. Herfkens and C. A. Taylor. Quantification of hemodynamics in abdominal aortic aneurysms during rest and exercise using magnetic resonance imaging and computational fluid dynamics. Annals of biomedical engineering, 38(4), 1288-1313, 2010

39. Lipton, Z. C., D. C. Kale, C. Elkan, and R. Wetzel. Learning to Diagnose with LSTM Recurrent Neural Networks. arXiv preprint 1511.03677, 2015.

40. Marcinkowska-Gapińska, A., J. Gapinski, W. Elikowski, F. Jaroszyk, and L. Kubisz. Comparison of three rheological models of shear flow behavior studied on blood samples from post-infarction patients. Medical and Biological Engineering and Computing 45:837$844,2007$.

41. Martufi, G., M. Lindquist Liljeqvist, N. Sakalihasan, G. Panuccio, R. Hultgren, J. Roy, and T. C. Gasser. Local Diameter, Wall Stress, and Thrombus Thickness Influence the Local Growth of Abdominal Aortic Aneurysms. Journal of Endovascular Therapy 23, 2016. 
42. McGloughlin, T. M. Biomechanics and Mechanobiology of Aneurysms, volume 7 of Studies in Mechanobiology, Tissue Engineering and Biomaterials, Berlin, Heidelberg: Springer Berlin Heidelberg2011, 1-33 pp.

43. Mills, C. J., I. T. Gabe, J. H. Gault, D. T. Mason, J. Ross, E. Braunwald, and J. P. Shillingford. Pressure-flow relationships and vascular impedance in man. Cardiovascular Research 4:405-417, 1970.

44. Moore, J., S. Maier, D. Ku, and P. Boesiger. Hemodynamics in the abdominal aorta: A comparison of in vitro and in vivo measurements. Journal of Applied Physiology 76:15201527, 1994.

45. Nagel, T., N. Resnick, C. F. Dewey, and M. A. Gimbrone. Vascular Endothelial Cells Respond to Spatial Gradients in Fluid Shear Stress by Enhanced Activation of Transcription Factors. Arteriosclerosis, Thrombosis, and Vascular Biology, 1999.

46. ORourke, M. J., J. P., McCullough, and S. Kelly. An investigation of the relationship between hemodynamics and thrombus deposition within patient-specific models of abdominal aortic aneurysm. Proceedings of the Institution of Mechanical Engineers, Part H: Journal of Engineering in Medicine, 226(7), 548-564, 2012.

47. Ouriel, K., R. M. Green, C. Donayre, C. K. Shortell, J. Elliott, and J. A. DeWeese. An evaluation of new methods of expressing aortic aneurysm size: Relationship to rupture. Journal of Vascular Surgery 15:12-20, 1992.

48. Parkinson, F., S. Ferguson, P. Lewis, I. M. Williams, C. P. Twine, and South East Wales Vascular Network. Rupture rates of untreated large abdominal aortic aneurysms in patients unfit for elective repair. Journal of Vascular Surgery 61:1606-1612, 2015.

49. Raghavan, M. L., J. Kratzberg, E. M. Castro de Tolosa, M. M. Hanaoka, P. Walker, and E. S. da Silva. Regional distribution of wall thickness and failure properties of human abdominal aortic aneurysm. Journal of Biomechanics 39:3010-3016, 2006. 
50. Reeps, C., A. Maier, J. Pelisek, F. Härtl, V. Grabher-Meier, W. A. Wall, M. Essler, H. H. Eckstein, and M. W. Gee. Measuring and modeling patient-specific distributions of material properties in abdominal aortic aneurysm wall. Biomechanics and Modeling in Mechanobiology 12:717-733, 2013.

51. Rowland, E. M., Y. Mohamied, K. Yean Chooi, E. L. Bailey, and P. D. Weinberg. Comparison of Statistical Methods for Assessing Spatial Correlations Between Maps of Different Arterial Properties. Journal of Biomechanical Engineering 137:101003, 2015.

52. P. J. Roache Perspective: A Method for Uniform Reporting of Grid Refinement Studies Journal of Fluids Engineering 116(3), 405-413, 1994)

53. Shadden, S. C. and S. Hendabadi. Potential fluid mechanic pathways of platelet activation. Biomechanics and Modeling in Mechanobiology 12:467-474, 2013.

54. Hansen, K. B., A. Arzani, and S. C. Shadden. Mechanical platelet activation potential in abdominal aortic aneurysms. Journal of biomechanical engineering, 137(4), 041005, 2015.

55. Shum, J., G. Martufi, E. Di Martino, C. B. Washington, J. Grisafi, S. C. Muluk, and E. A. Finol. Quantitative assessment of abdominal aortic aneurysm geometry. Annals of Biomedical Engineering 39:277-286, 2011.

56. Shum, J., A. Xu, I. Chatnuntawech, and E. A. Finol. A framework for the automatic generation of surface topologies for abdominal aortic aneurysm models. Annals of Biomedical Engineering 39:249-259, 2011.

57. Silverberg, E., C. C. Boring, and T. S. Squires. Cancer statistics, 1990. CA: A Cancer Journal for Clinicians 40:9-26, 1990.

58. Stenbaek, J., B. Kalin, and J. Swedenborg. Growth of thrombus may be a better predictor of rupture than diameter in patients with abdominal aortic aneurysms. European Journal of Vascular and Endovascular Surgery 20:466-469, 2000. 
59. Solberg, S., Singh, K., Wilsgaard, T., and Jacobsen, B. K. Increased growth rate of abdominal aortic aneurysms in women. The Troms study.. EEuropean journal of vascular and endovascular surgery, 29(2), 145-149,2005

60. Stevens, R. R., A. Grytsan, J. Biasetti, J. Roy, M. L. Liljeqvist, and T. Christian Gasser. Biomechanical changes during abdominal aortic aneurysm growth. PLoS ONE 12:e0187421, 2017.

61. Strub, F., H. De Vries, J. Mary, B. Piot, A. Courvile, and O. Pietquin. End-to-end optimization of goal-driven and visually grounded dialogue systems. IJCAI International Joint Conference on Artificial Intelligence 13:2765-2771, 2017.

62. Tanios, F., M. W. Gee, J. Pelisek, S. Kehl, J. Biehler, V. Grabher-Meier, W. A. Wall, H. H. Eckstein, and C. Reeps. Interaction of Biomechanics with Extracellular Matrix Components in Abdominal Aortic Aneurysm Wall. European Journal of Vascular and Endovascular Surgery 50:167-174, 2015.

63. The Theano Development Team, R. Al-Rfou, G. Alain, A. Almahairi, C. Angermueller, D. Bahdanau, N. Ballas, F. Bastien, J. Bayer, A. Belikov, A. Belopolsky, Y. Bengio, A. Bergeron, J. Bergstra, V. Bisson, J. B. Snyder, N. Bouchard, N. BoulangerLewandowski, X. Bouthillier, A. de Brébisson, O. Breuleux, P.-L. Carrier, K. Cho, J. Chorowski, P. Christiano, T. Cooijmans, M.-A. Côté, M. Côté, A. Courville, Y. N. Dauphin, O. Delalleau, J. Demouth, G. Desjardins, S. Dieleman, L. Dinh, M. Ducoffe, V. Dumoulin, S. E. Kahou, D. Erhan, Z. Fan, O. Firat, M. Germain, X. Glorot, I. Goodfellow, M. Graham, C. Gulcehre, P. Hamel, I. Harlouchet, J.-P. Heng, B. Hidasi, S. Honari, A. Jain, S. Jean, K. Jia, M. Korobov, V. Kulkarni, A. Lamb, P. Lamblin, E. Larsen, C. Laurent, S. Lee, S. Lefrancois, S. Lemieux, N. Léonard, Z. Lin, J. A. Livezey, C. Lorenz, J. Lowin, Q. Ma, P.-A. Manzagol, O. Mastropietro, R. T. McGibbon, R. Memisevic, B. van Merriënboer, V. Michalski, M. Mirza, A. Orlandi, C. Pal, R. Pascanu, M. Pezeshki, C. Raffel, D. Renshaw, M. Rocklin, A. Romero, M. Roth, P. Sadowski, J. Salvatier, F. Savard, J. Schlüter, J. Schulman, G. Schwartz, I. V. Serban, D. Serdyuk, S. Shabanian, C. Simon, 
S. Spieckermann, S. R. Subramanyam, J. Sygnowski, J. Tanguay, G. van Tulder, J. Turian, S. Urban, P. Vincent, F. Visin, H. de Vries, D. Warde-Farley, D. J. Webb, M. Willson, K. Xu, L. Xue, L. Yao, S. Zhang, and Y. Zhang. Theano: A Python framework for fast computation of mathematical expressions. arXiv e-prints p. 19, 2016.

64. Tzirakis, K., Y. Kamarianakis, E. Metaxa, N. Kontopodis, C. V. Ioannou, and Y. Papaharilaou. A robust approach for exploring hemodynamics and thrombus growth associations in abdominal aortic aneurysms. Medical and Biological Engineering and Computing $55: 1493-1506,2017$.

65. Vergara, C., D. Le Van, M. Quadrio, L. Formaggia, and M. Domanin. Large eddy simulations of blood dynamics in abdominal aortic aneurysms. Medical engineering \& physics, 47, 38-46, 2017

66. Womersley, J. R. Method for the calculation of velocity, rate of flow and viscous drag in arteries when the pressure gradient is known. The Journal of Physiology 127:553-563, 1955.

67. Wu, J. and S. C. Shadden. Coupled Simulation of Hemodynamics and Vascular Growth and Remodeling in a Subject-Specific Geometry. Annals of biomedical engineering, 43(7), 1543-1554.

68. Xiao, N., J. D. Humphrey, and C. A. Figueroa. Multi-scale computational model of three-dimensional hemodynamics within a deformable full-body arterial network. Journal of Computational Physics 244:22-40, 2013.

69. Xu, X., B. Wang, C. Ren, J. Hu, D. A. Greenberg, T. Chen, L. Xie, and K. Jin. Agerelated Impairment of Vascular Structure and Functions. Aging and disease 8:590-610, 2017.

70. Yushkevich, P. A., J. Piven, H. C. Hazlett, R. G. Smith, S. Ho, J. C. Gee, and G. Gerig. User-guided 3D active contour segmentation of anatomical structures: Significantly improved efficiency and reliability. NeuroImage 31:1116-1128, 2006. 
71. Zambrano, B. A., H. Gharahi, C. Y. Lim, F. A. Jaberi, J. Choi, W. Lee, and S. Baek. Association of Intraluminal Thrombus, Hemodynamic Forces, and Abdominal Aortic Aneurysm Expansion Using Longitudinal CT Images. Annals of Biomedical Engineering 44:1502-1514, 2016.

\section{Electronic Supplementary Material}

\subsection{Boundary conditions}

[Figure 9 about here.]

[Table 8 about here.]

\subsection{Metrics description}

\subsection{Local distribution}

[Table 9 about here.]

(1)

[Figure 10 about here.]

[Figure 11 about here.]

[Figure 12 about here.]

[Figure 13 about here.]

[Figure 14 about here.] 


\section{List of Figures}

1 Overview of the study. . . . . . . . . . . . . . .

2 Patching process of the lumen surface. Left: centerline of the vessel lumen, Center: circumferential discretization, colored by the angular index. Right: centerline-based longitudinal discretization colored by the abscissa along the centerline. . . . . . . . . . . . . . . . . . . .

3 Aorta of the 9 healthy patients included in the study as control subjects. Most present various degrees of tortuosity, due to arteries aging. . . . . . . . .

4 Example of different growth dynamics on two patients. The segmented lumen is in red, while ILT is transparent. . . . . . . . . . . . . . . . . . .

5 Evolution with time of selected parameters among patients. AAA ending up as high-risk are represented by red lines while low-risk AAA are in blue. Averaged behaviours of the two groups are in bold color. . . . . . . . . . . . 39

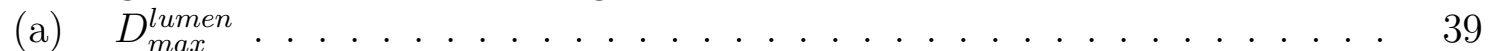

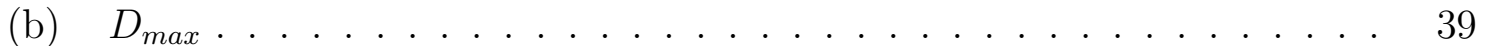

(c) Lumen centerline tortuosity . . . . . . . . . . . . . . . . . . . . 39

(d) Lumen NSI . . . . . . . . . . . . . . . . . . . . . . . . 39

6 a and c: Bivariate distributions and kernel density plot of ECAP $\left(\mathrm{Pa}^{-1}\right)$ versus the normalized distance from the lumen wall to the centerline of two simulations from two different patients. b and c: Distribution plots of the Spearmans's $\rho$ from the bootstrap evaluations. The top and bottom cases illustrate the variety of the bivariate distributions and correlations encountered in the study.

7 Boxplot of the distribution of Spearman's $\rho$ between local flow and morphological evaluation metrics. On the left, all scans are evaluated separately and on the right statistics are patient-wise. The boxes represent the inter-quartile range (IQR) i.e. data between the 25 (Q1) and 75\% (Q3) percentile. Bottom whisker is $\mathrm{Q} 1-1.5 \mathrm{IQR}$ and top whisker is $\mathrm{Q} 3+1.5 \mathrm{IQR}$. Outliers are not represented for readability. Correlations are computed on patch-wise data for each scan. The large dispersion of Spearman's $\rho$ distribution is illustrated in Figure 6 for one metric. . . . . . . . . . . . . . . . . . . . .

8 Left: ROC curves for the classification of high risk (i.e. risk predictor). Right: Top 10 features ranked with respect to predictability of the target variable. The gray area represents the standard deviation ( \pm 1 st. dev.) of all ROC generated during the repeated cross-validation process. . . . . . . . . . . . 42

I Volumetric flow rate imposed at the inlet of the AAA . . . . . . . . . . . .

II View of patched metrics on an aneurysm. From left to right and top to bottom: lumen with ILT overlaid, $H^{I L T}[\mathrm{~mm}]$, ILT coverage [.], local distance to the centerline $[\mathrm{mm}]$, OSI [.], TAWSS [Pa], ECAP $\left[\mathrm{Pa}^{-1}\right]$ and RRT $\left[\mathrm{Pa}^{-1}\right]$. The displayed AAA is also visible in Figure V and Figure VI . . . . . . . . 44

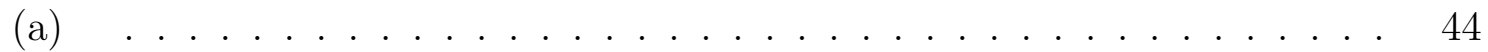

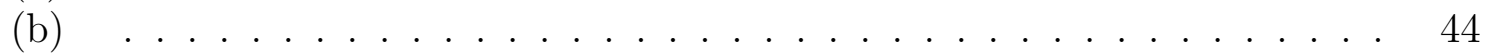

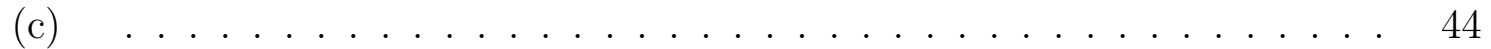

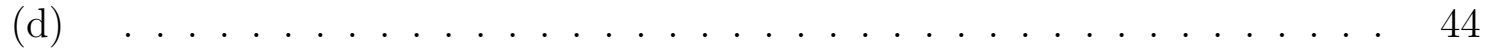


(e)

(h)

III Boxplot of local distribution of various metrics, for all patient, along their follow-up. Statistical distribution is built from data from all 600 patches. The box represents the inter-quartile range (IQR) or data between the 25 (Q1) and $75 \%$ (Q3) percentile. Bottom whisker is Q1 - 1.5IQR and top whisker is $\mathrm{Q} 3+$ 1.5IQR. Outliers are not represented for the sake of readability. . . . .

IV View of local distance $(\mathrm{mm})$ from the lumen to the centerline mapped on the lumen and averaged on patches for patient 6 (a), 22 (b) and 21 (c). Patient 6 exhibits a strong and localized growth of the lumen. Patient $21 \mathrm{~s}$ lumen is pretty tubular with a constant diameter while patient 22 's diameter is healthy at the proximal neck and over $50 \mathrm{~mm}$ at the $D_{\max }$ location, hence the large dispersion of values seen on the boxplot. . . . . . . . . . . . . . . . . 46

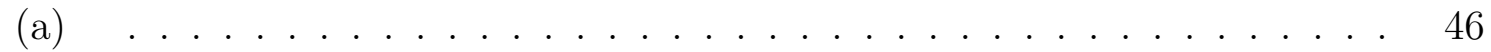

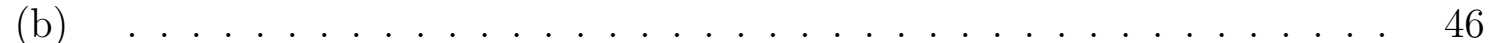

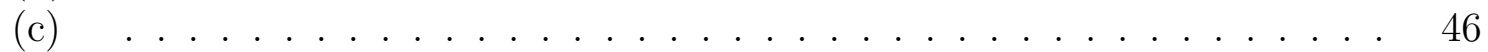

V For 4 patients, RRT averaged on patches. Patient 10 (a), exhibits a steady growth with time. Patient 28 (b): RRT standard deviation is relatively small compared to patient 29 (c). For patient 15 (d), RRT decreases before increasing. 47

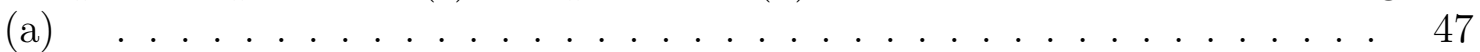

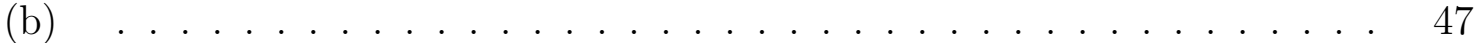

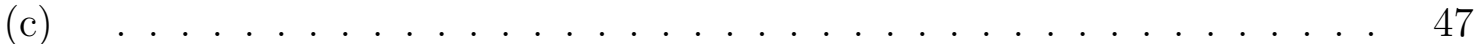

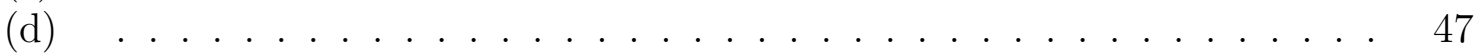

VI ECAP averaged on patches, for 4 patients. ECAP, similarly to the RRT contains information from OSI and TAWSS. Patient 10 (a), monotonic growth, patient 28 (b) with a small standard deviation compared to patient 29 (c). Patient 15 (d) ECAP average, decreases and then increases. . . . . . . . . . 48

(a) . . . . . . . . . . . . . . . . . . . . . . . 48

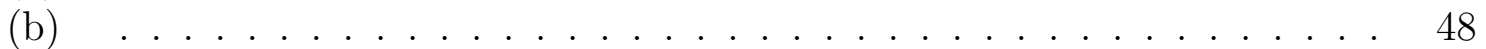

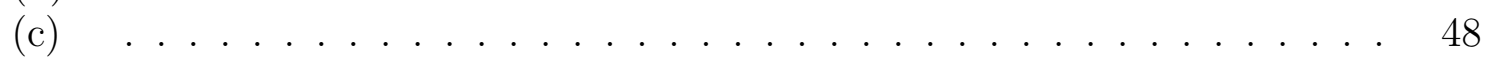

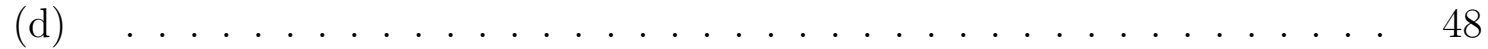




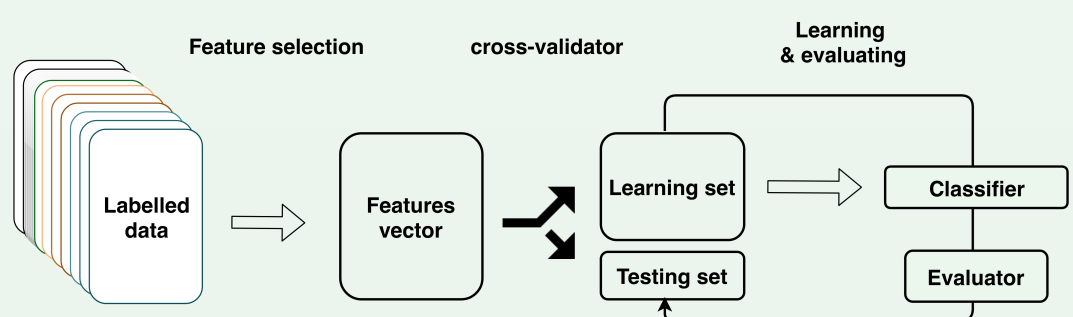

AAA risk prediction
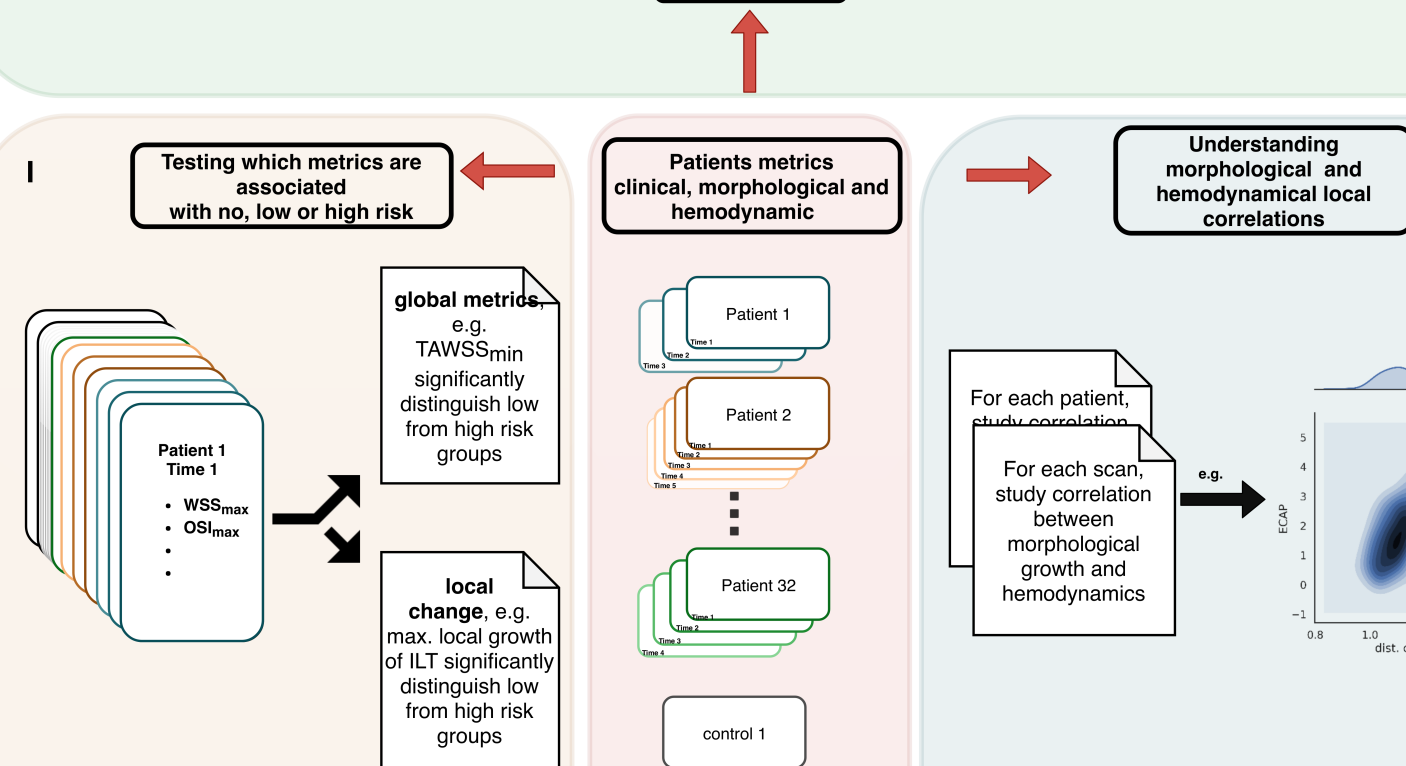
corremical loca

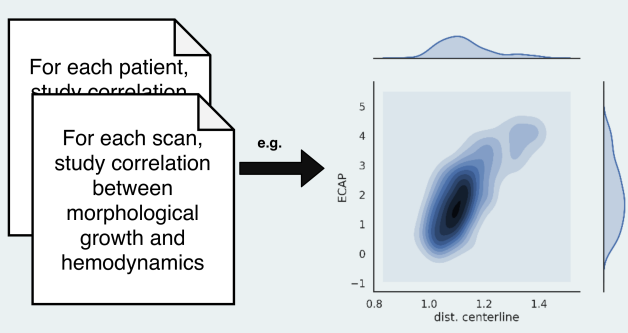

Figure 1: Overview of the study. 

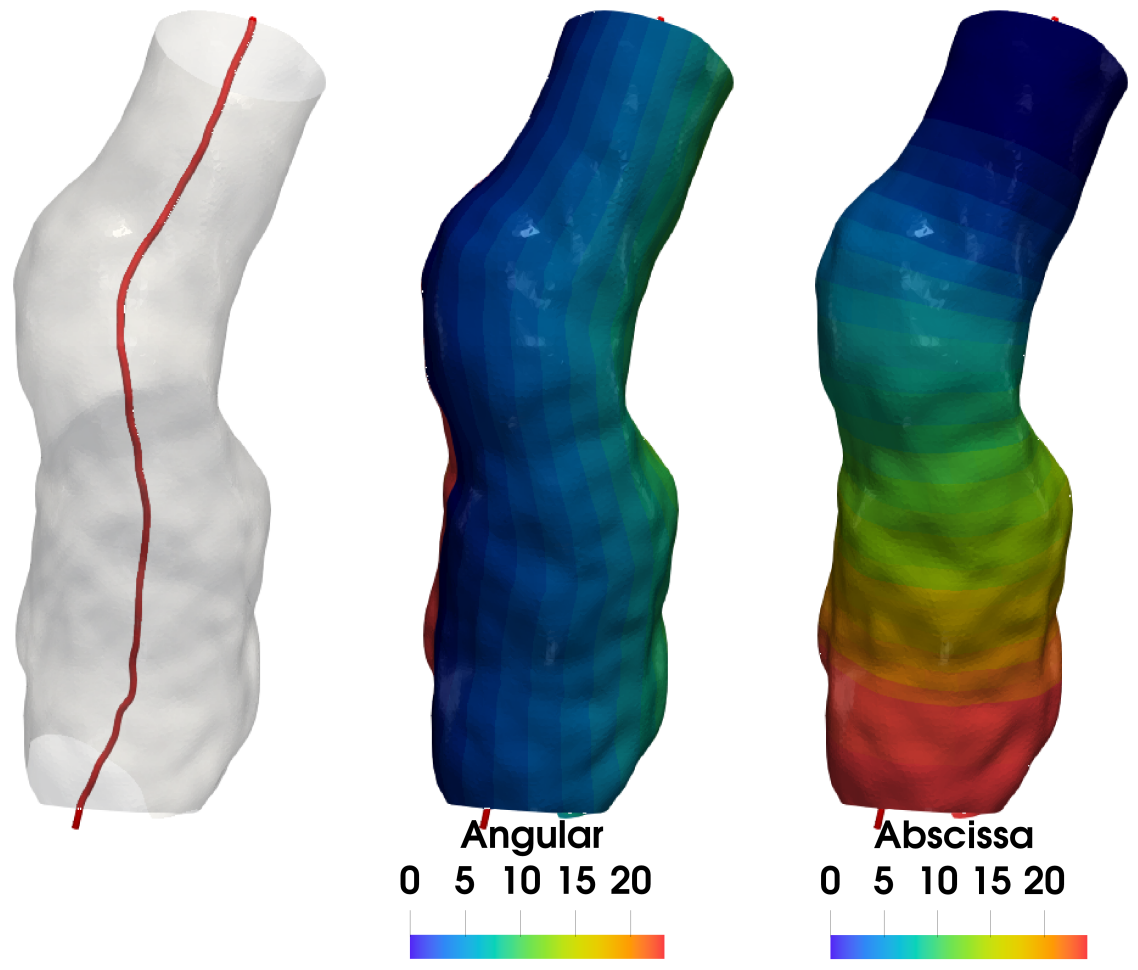

Figure 2: Patching process of the lumen surface. Left: centerline of the vessel lumen, Center: circumferential discretization, colored by the angular index. Right: centerline-based longitudinal discretization colored by the abscissa along the centerline. 


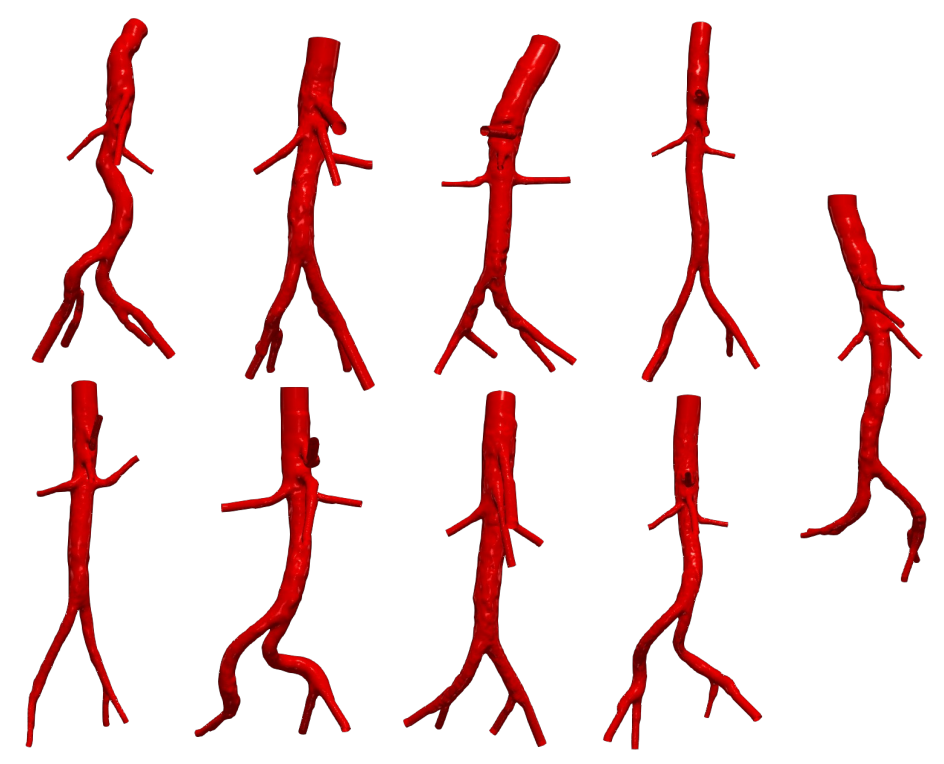

Figure 3: Aorta of the 9 healthy patients included in the study as control subjects. Most present various degrees of tortuosity, due to arteries aging. 


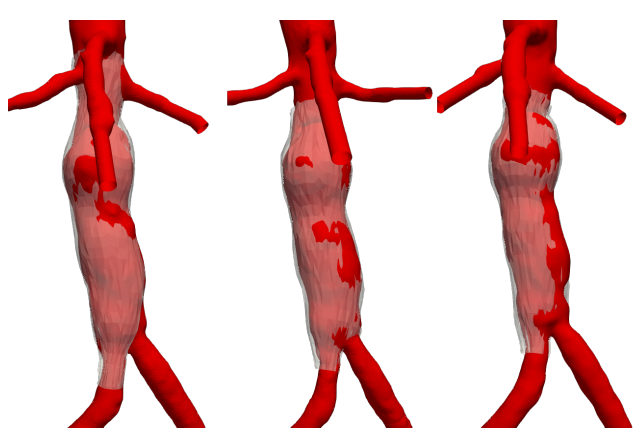

(a)

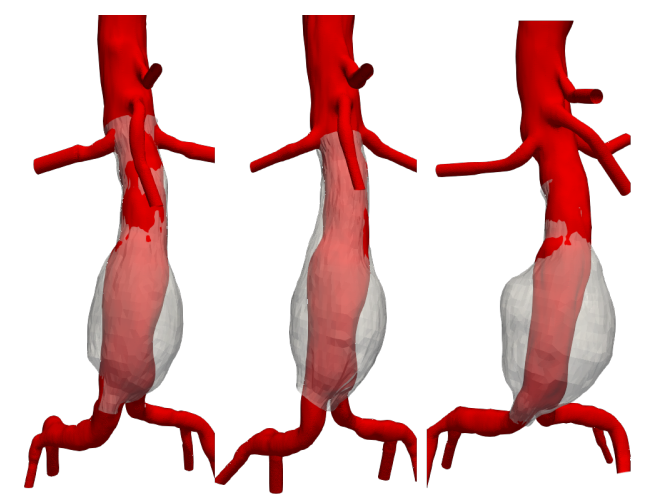

(b)

Figure 4: Example of different growth dynamics on two patients. The segmented lumen is in red, while ILT is transparent. 


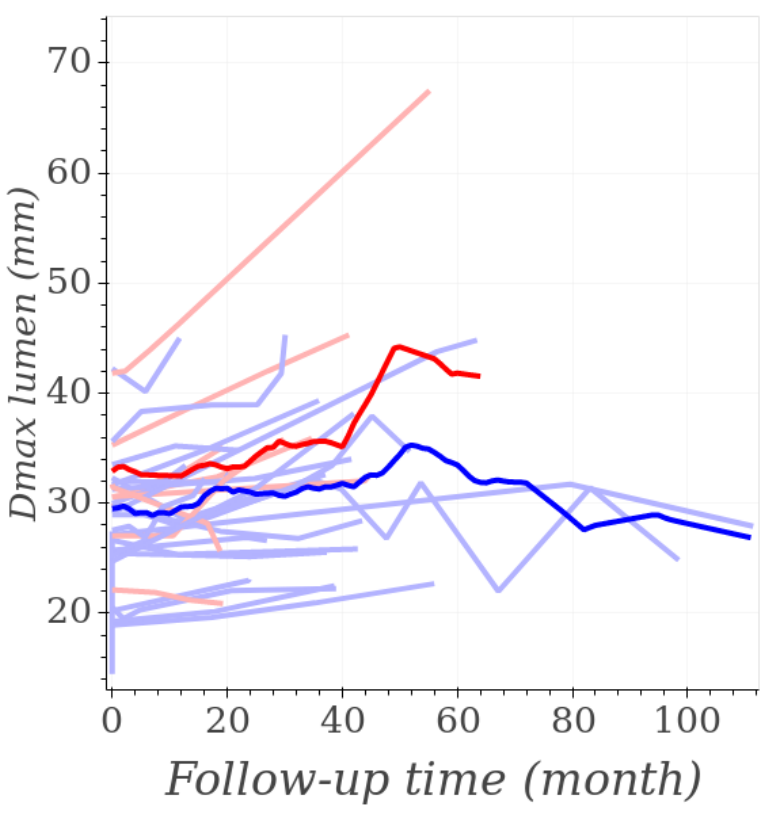

(a) $D_{\max }^{\text {lumen }}$

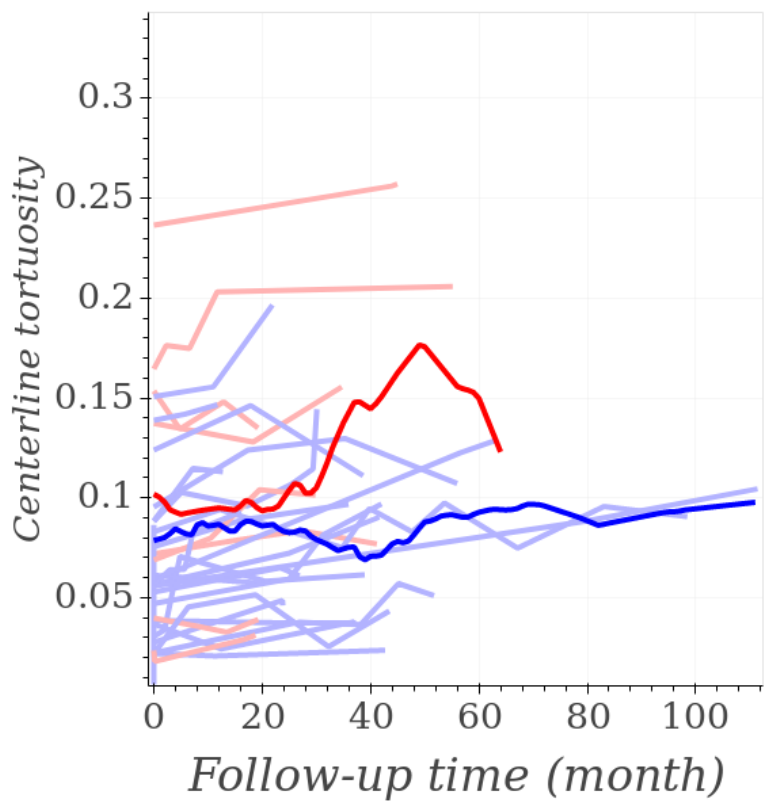

(c) Lumen centerline tortuosity

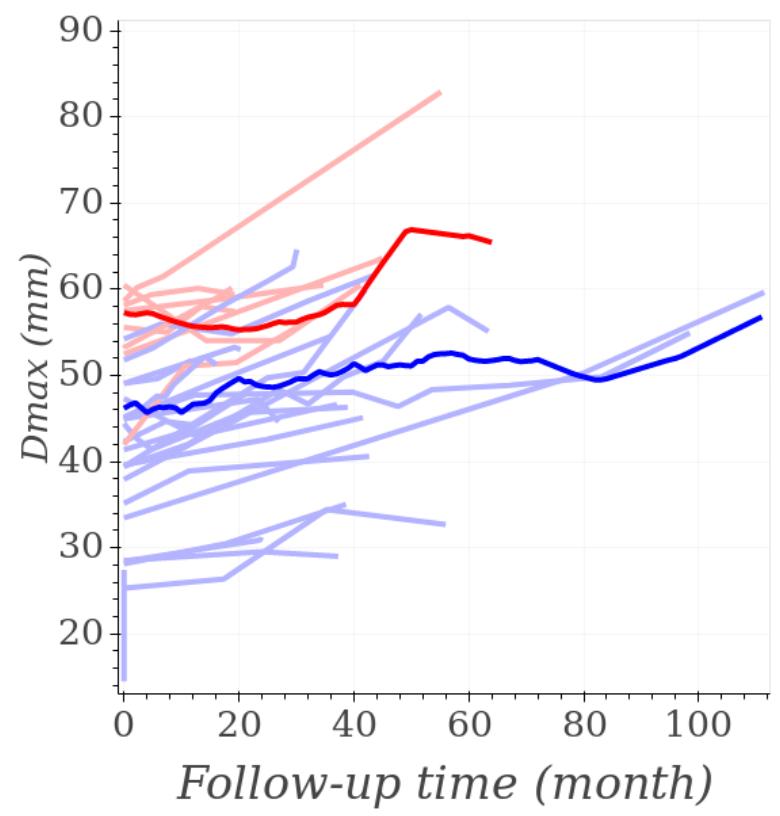

(b) $D_{\max }$

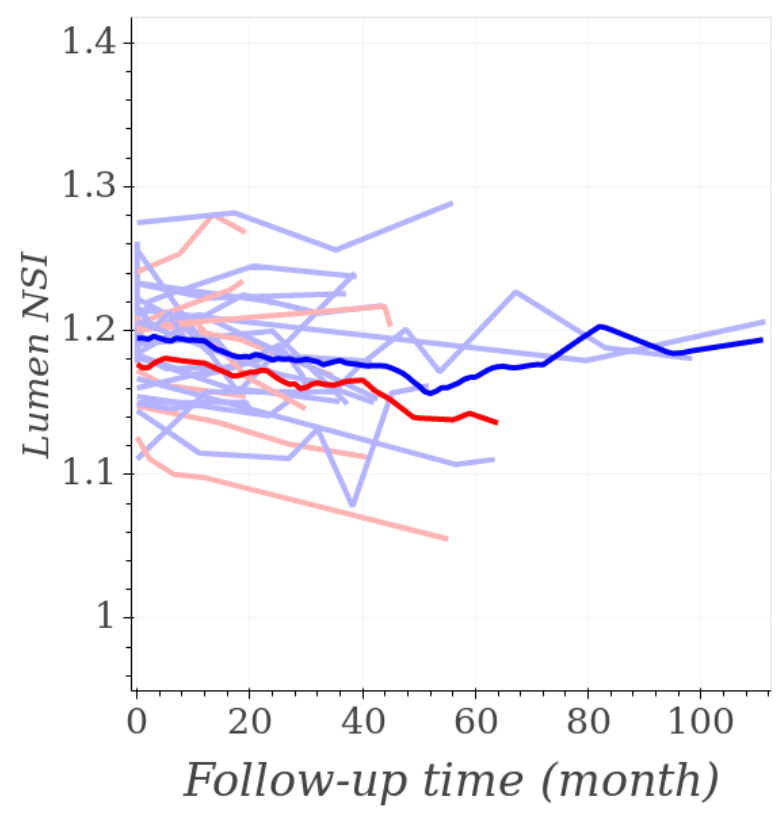

(d) Lumen NSI

Figure 5: Evolution with time of selected parameters among patients. AAA ending up as high-risk are represented by red lines while low-risk AAA are in blue. Averaged behaviours of the two groups are in bold color. 


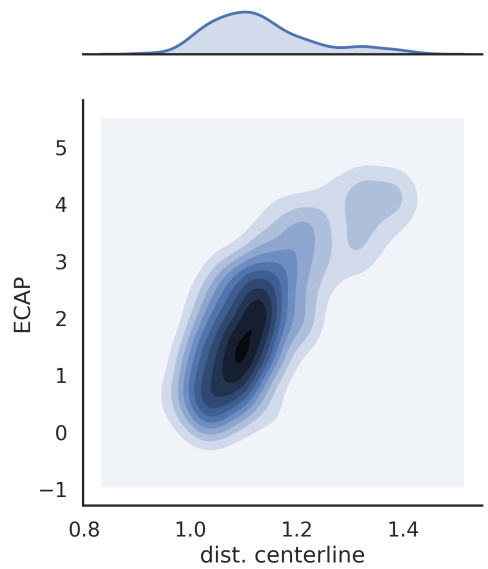

(a)

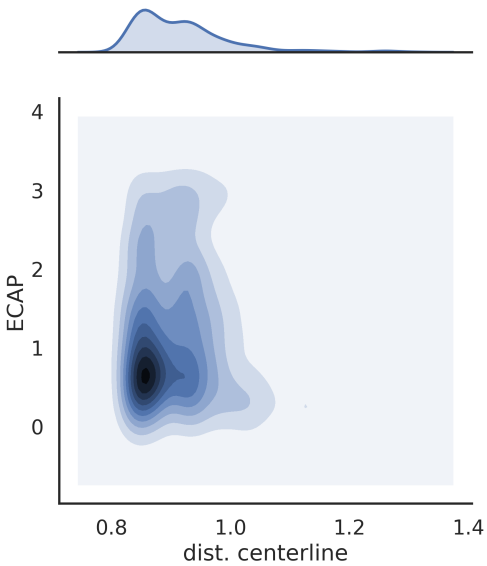

(c)

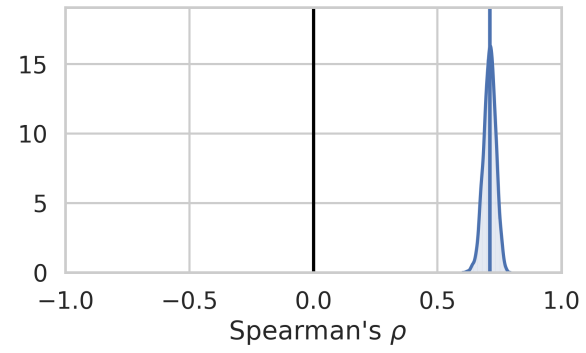

(b)

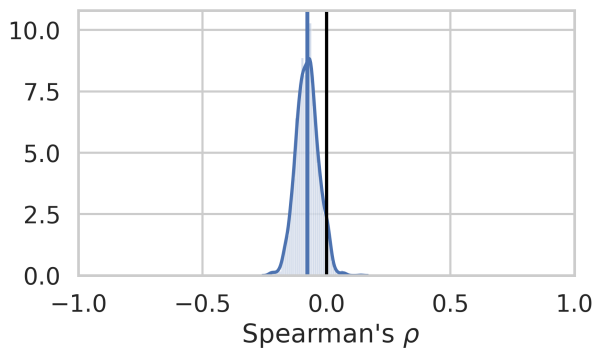

$(\mathrm{d})$

Figure 6: a and c: Bivariate distributions and kernel density plot of ECAP $\left(\mathrm{Pa}^{-1}\right)$ versus the normalized distance from the lumen wall to the centerline of two simulations from two different patients. b and c: Distribution plots of the Spearmans's $\rho$ from the bootstrap evaluations. The top and bottom cases illustrate the variety of the bivariate distributions and correlations encountered in the study. 


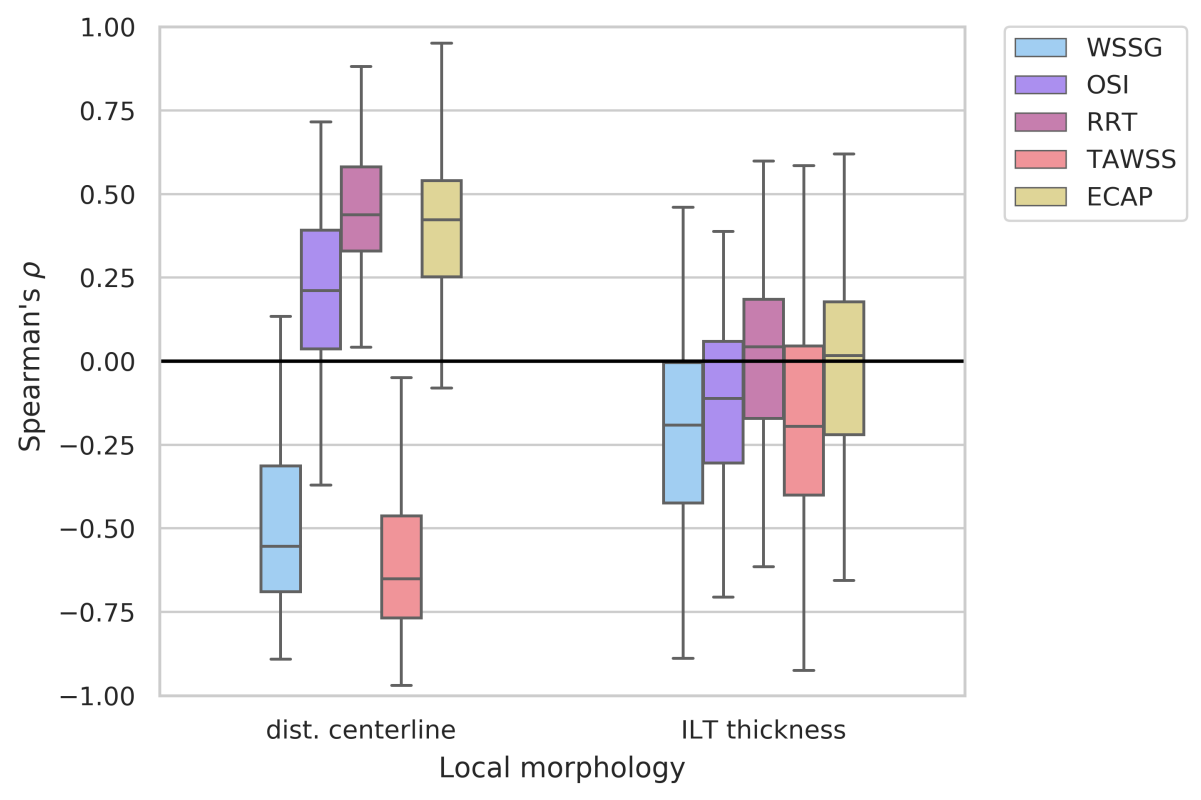

(a) All CTs evaluated independently.

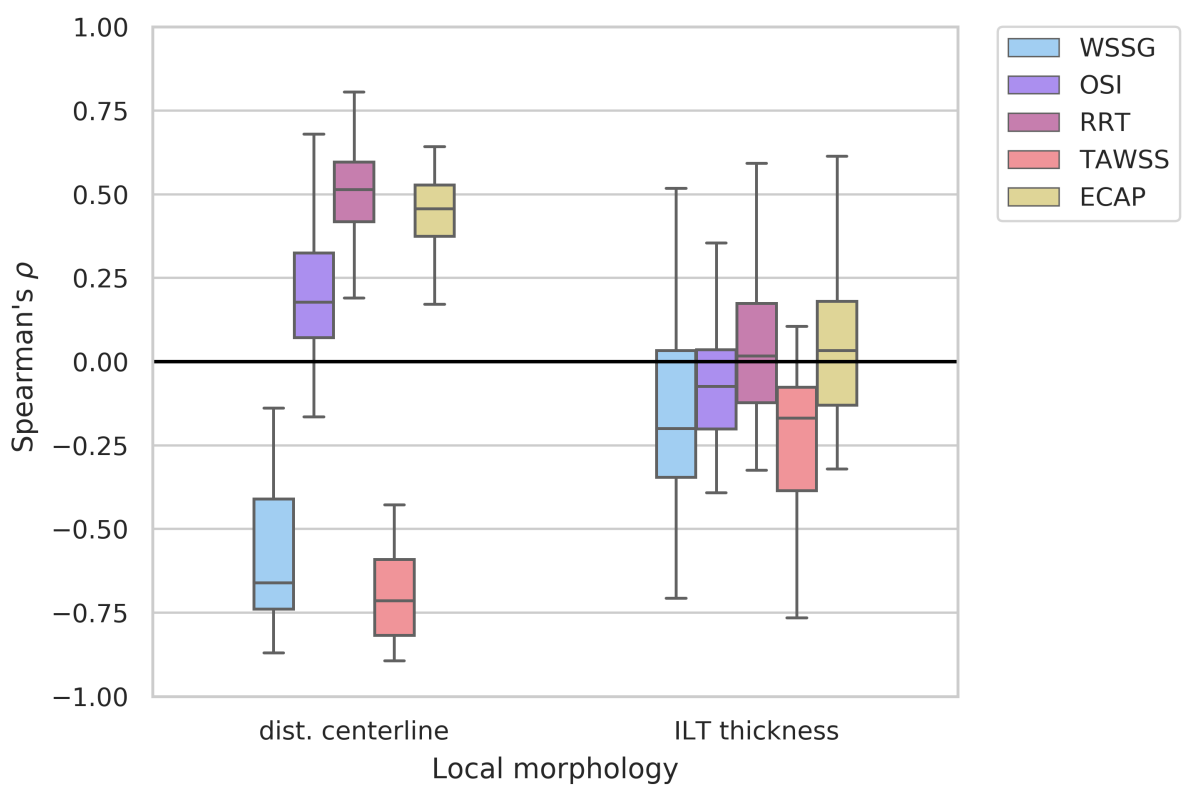

\section{(b) Statistics performed on each patient, (one correlation for all scans of that patient).}

Figure 7: Boxplot of the distribution of Spearman's $\rho$ between local flow and morphological evaluation metrics. On the left, all scans are evaluated separately and on the right statistics are patient-wise. The boxes represent the inter-quartile range (IQR) i.e. data between the 25 (Q1) and $75 \%$ (Q3) percentile. Bottom whisker is Q1 - 1.5IQR and top whisker is Q3 + 1.5IQR. Outliers are not represented for readability. Correlations are computed on patch-wise data for each scan. The large dispersion of Spearman's $\rho$ distribution is illustrated in Figure 6 for one metric. 
$\mathrm{D}_{\max }$

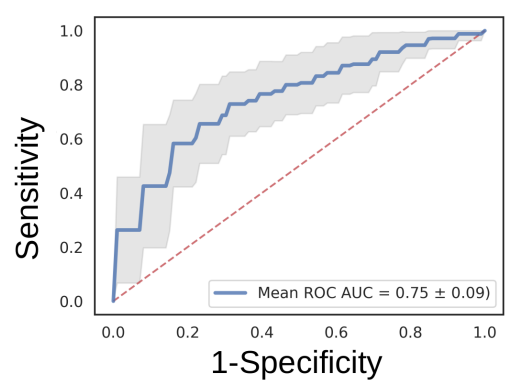

Clinical features
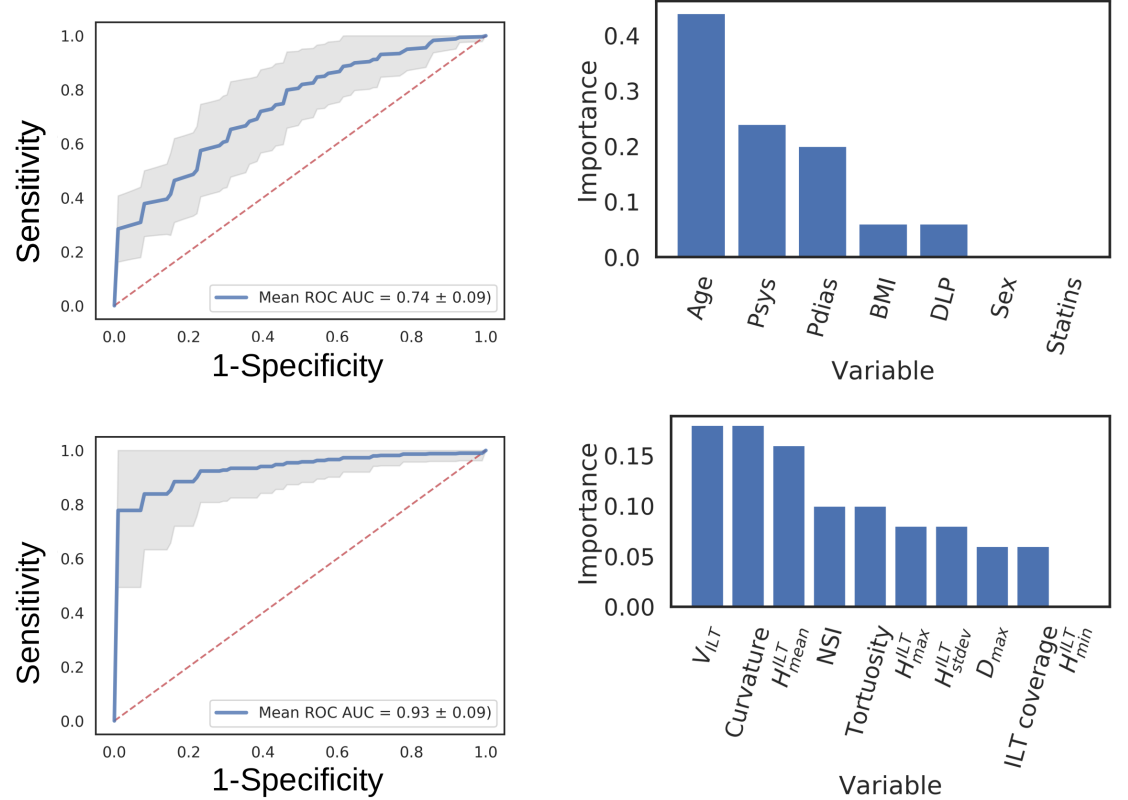

Morphological features
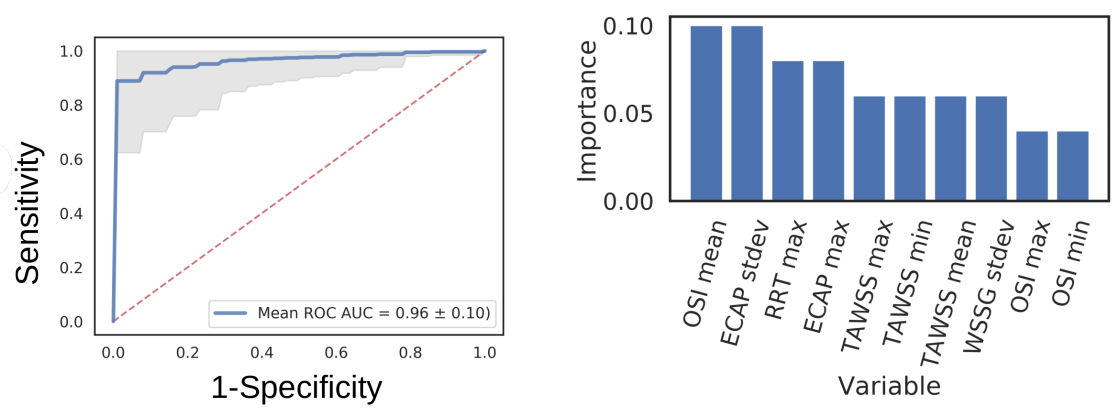

Hemodynamic features
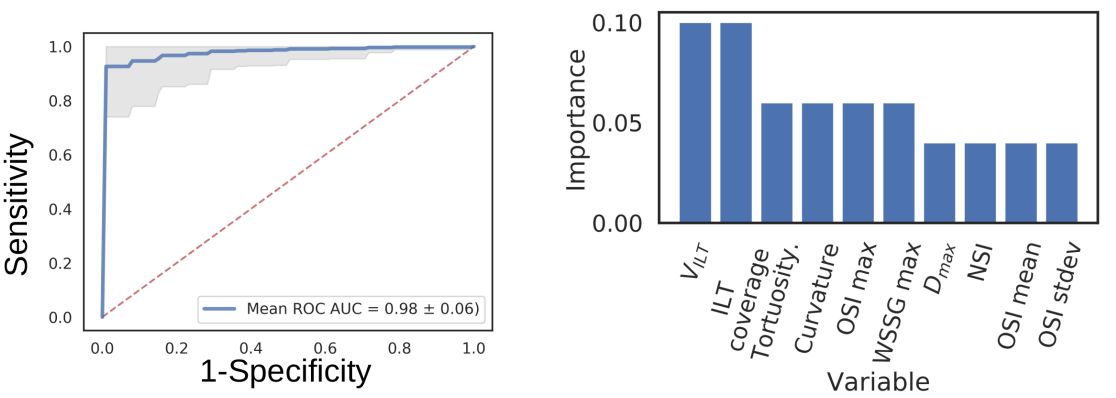

Figure 8: Left: ROC curves for the classification of high risk (i.e. risk predictor). Right: Top 10 features ranked with respect to predictability of the target variable. The gray area represents the standard deviation $( \pm 1$ st. dev.) of all ROC generated during the repeated cross-validation process. 


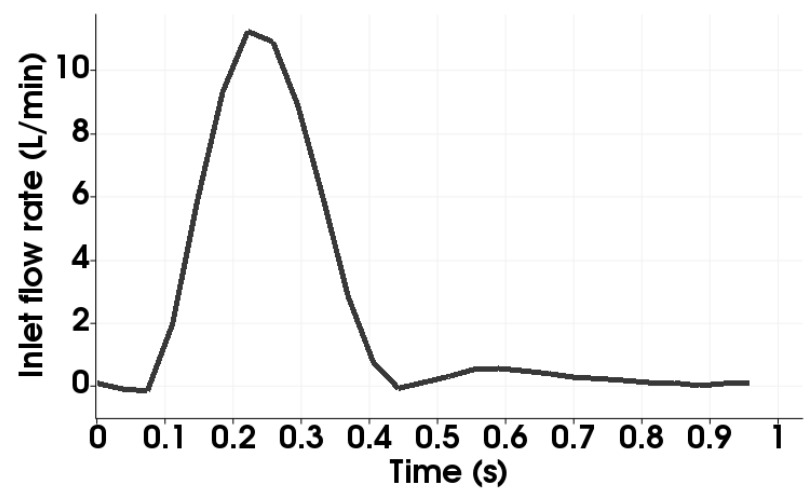

Figure I: Volumetric flow rate imposed at the inlet of the AAA. 


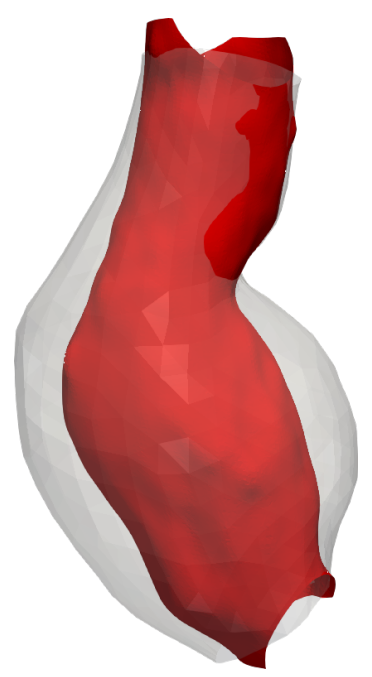

(a)

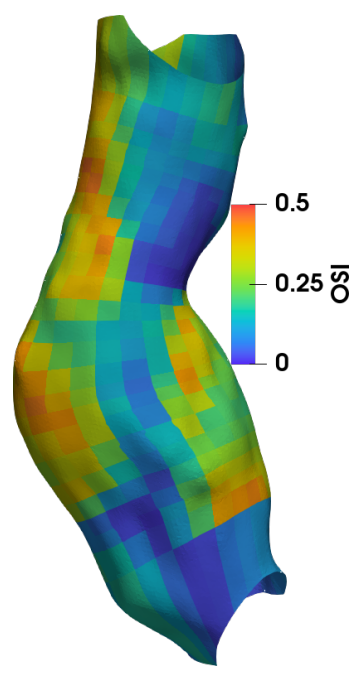

(e)

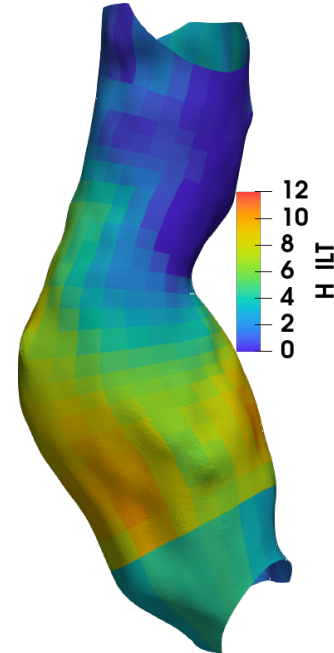

(b)

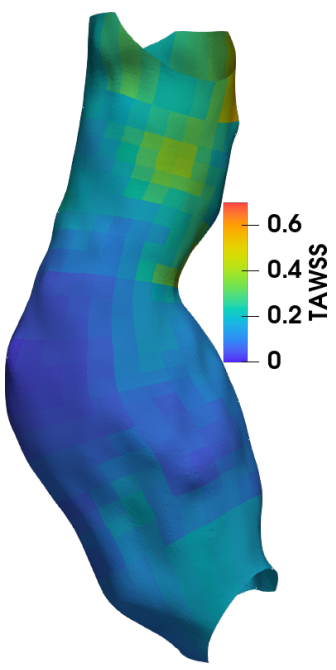

(f)

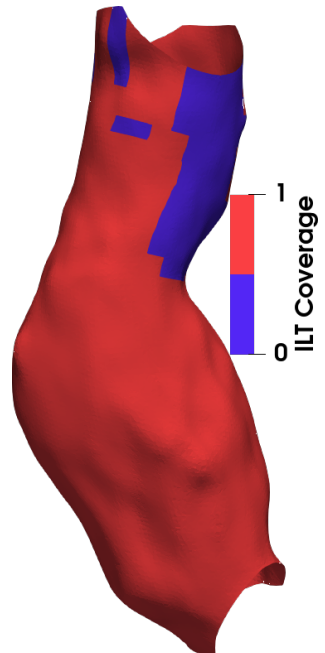

(c)

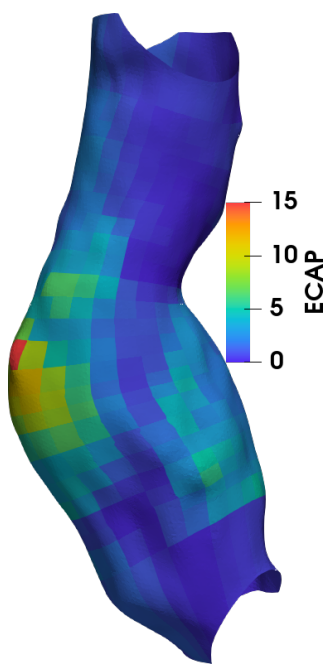

(g)

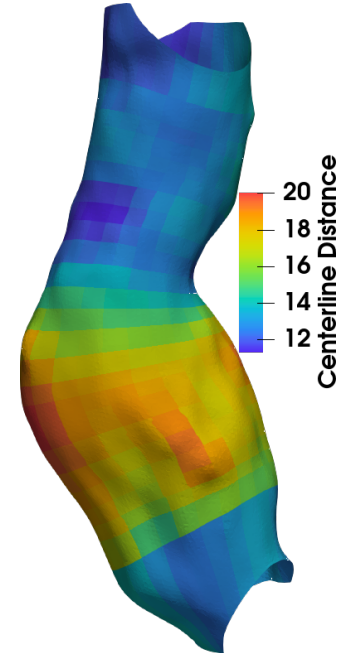

(d)

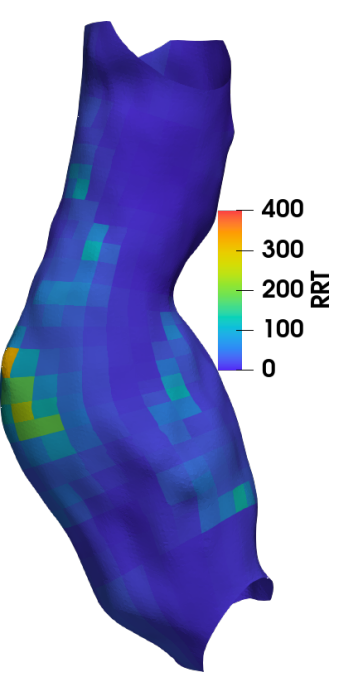

(h)

Figure II: View of patched metrics on an aneurysm. From left to right and top to bottom: lumen with ILT overlaid, $H^{I L T}[\mathrm{~mm}]$, ILT coverage [.], local distance to the centerline $[\mathrm{mm}]$, OSI [.], TAWSS $[\mathrm{Pa}]$, ECAP $\left[\mathrm{Pa}^{-1}\right]$ and RRT $\left[\mathrm{Pa}^{-1}\right]$. The displayed AAA is also visible in Figure V and Figure VI 


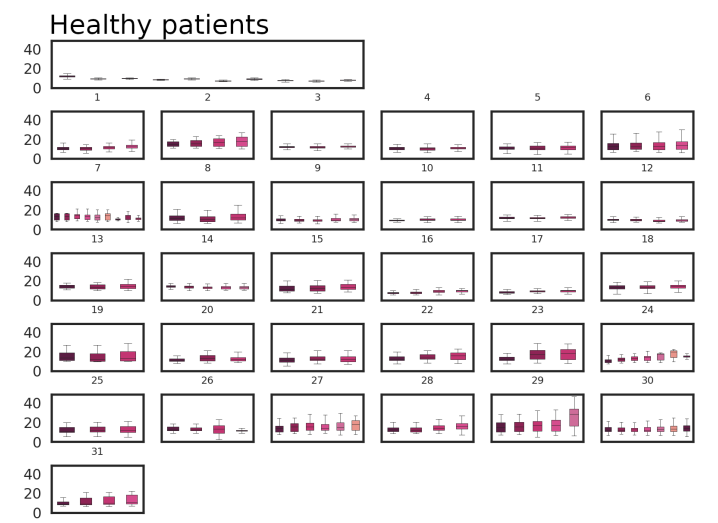

a) lumen - centerline distance

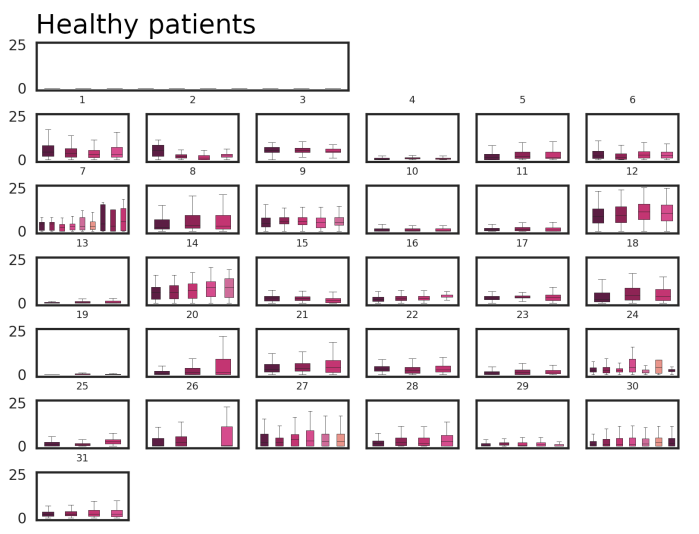

c) $H_{\max }^{\mathrm{ILT}}$

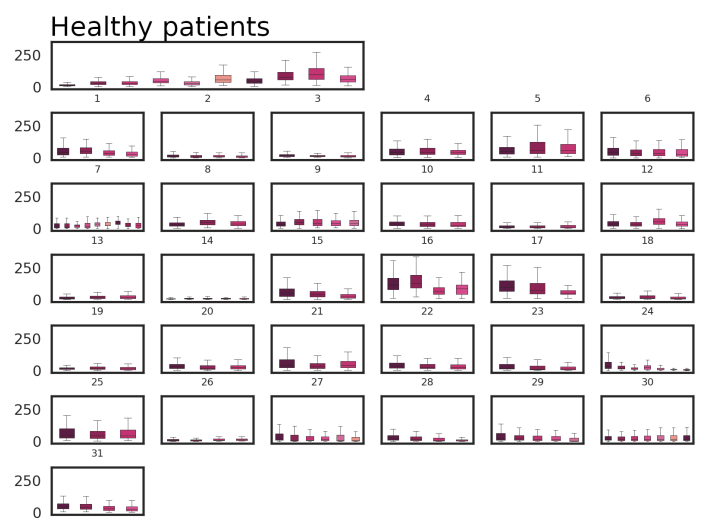

e) WSSG

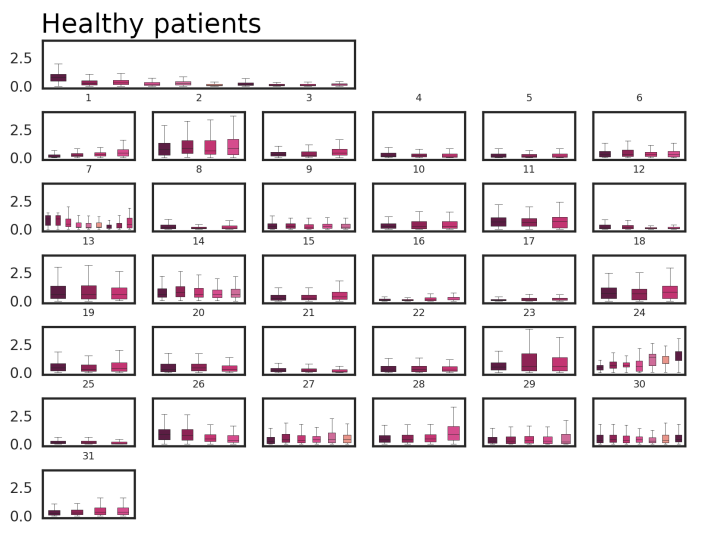

b) RRT

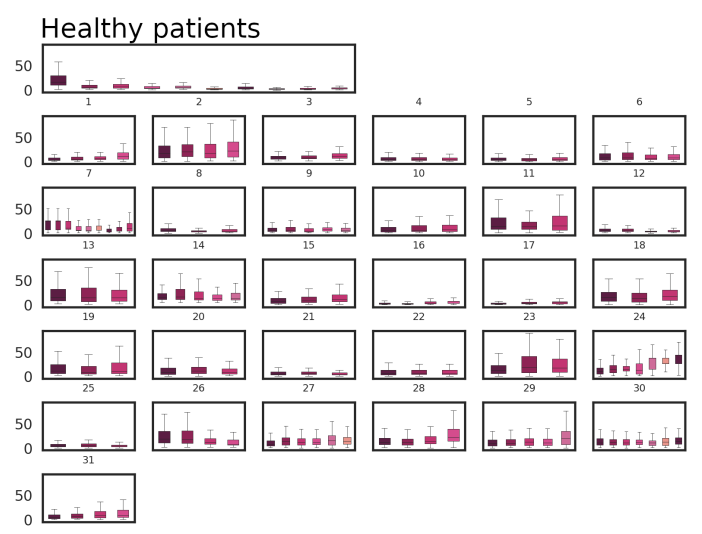

d) ECAP

Figure III: Boxplot of local distribution of various metrics, for all patient, along their followup. Statistical distribution is built from data from all 600 patches. The box represents the inter-quartile range (IQR) or data between the 25 (Q1) and 75\% (Q3) percentile. Bottom whisker is $\mathrm{Q} 1-1.5 \mathrm{IQR}$ and top whisker is $\mathrm{Q} 3+1.5 \mathrm{IQR}$. Outliers are not represented for the sake of readability. 


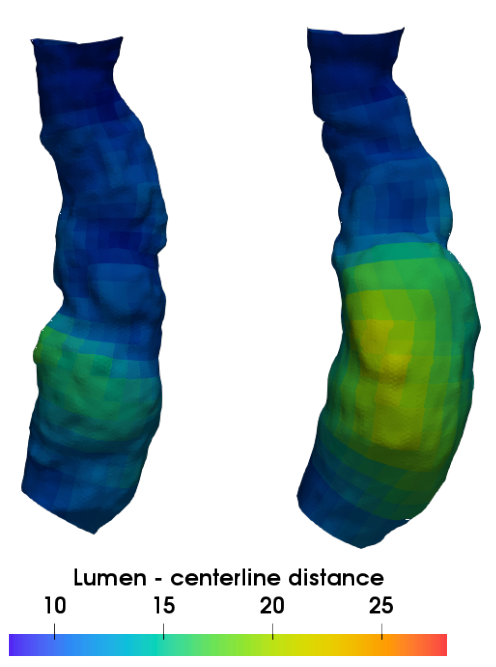

(a)
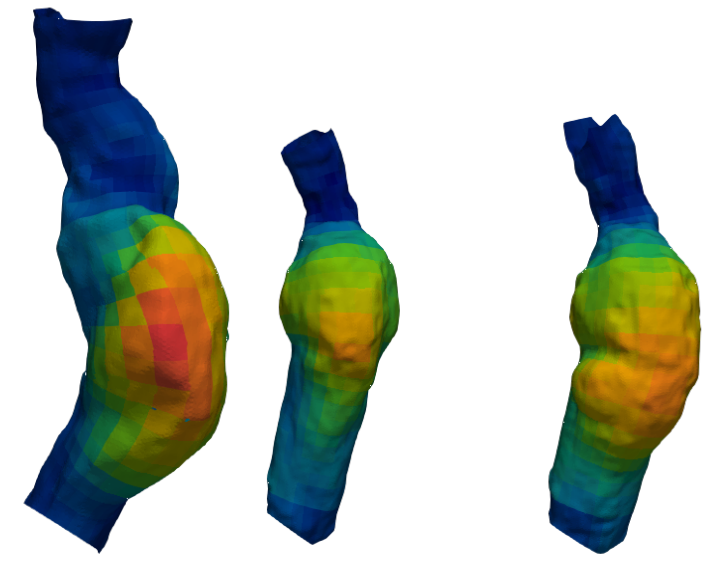

Lumen - centerline distance
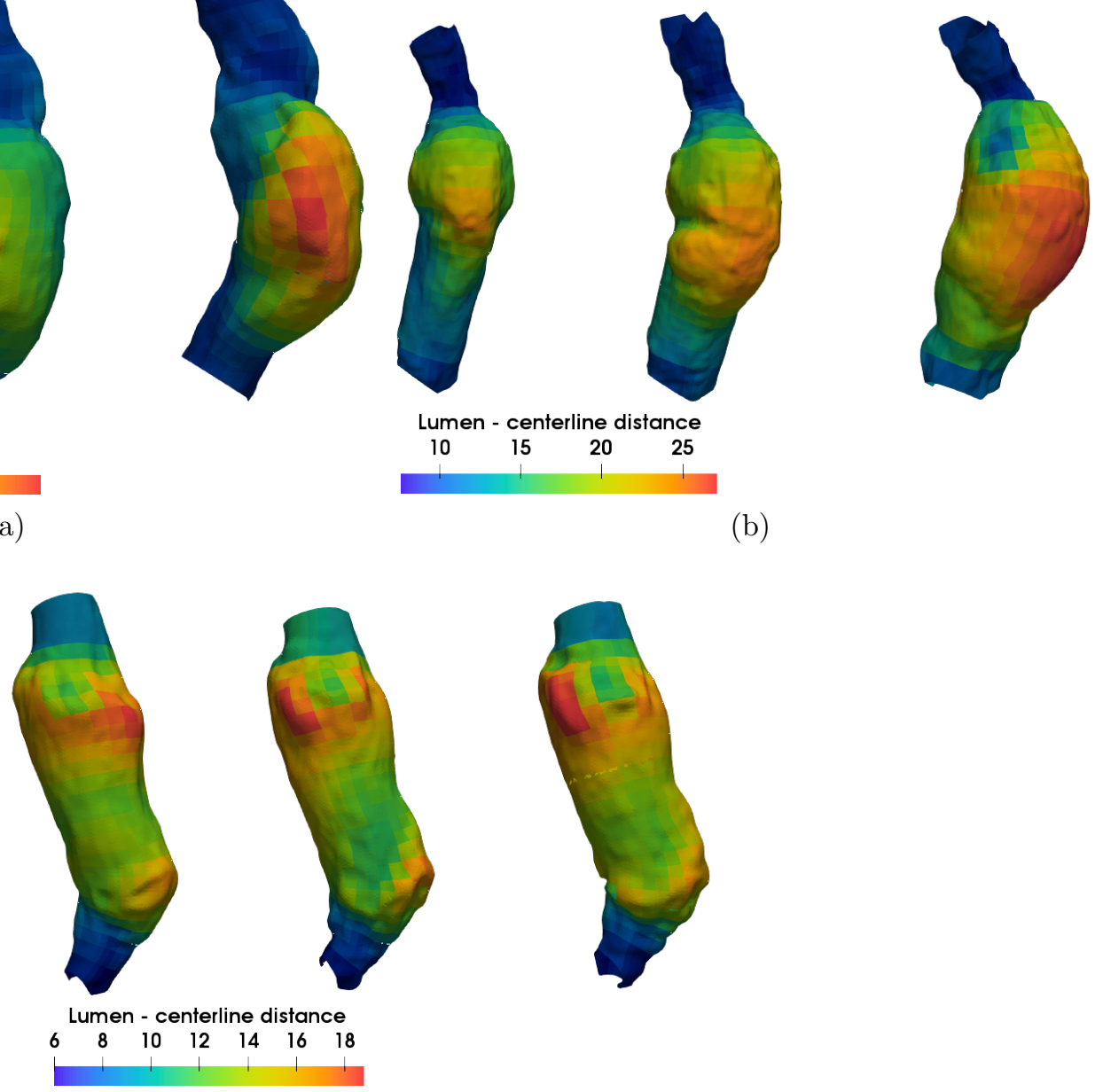

(b)

(c)

Figure IV: View of local distance $(\mathrm{mm})$ from the lumen to the centerline mapped on the lumen and averaged on patches for patient 6 (a), 22 (b) and 21 (c). Patient 6 exhibits a strong and localized growth of the lumen. Patient 21s lumen is pretty tubular with a constant diameter while patient 22's diameter is healthy at the proximal neck and over 50 $\mathrm{mm}$ at the $D_{\max }$ location, hence the large dispersion of values seen on the boxplot. 

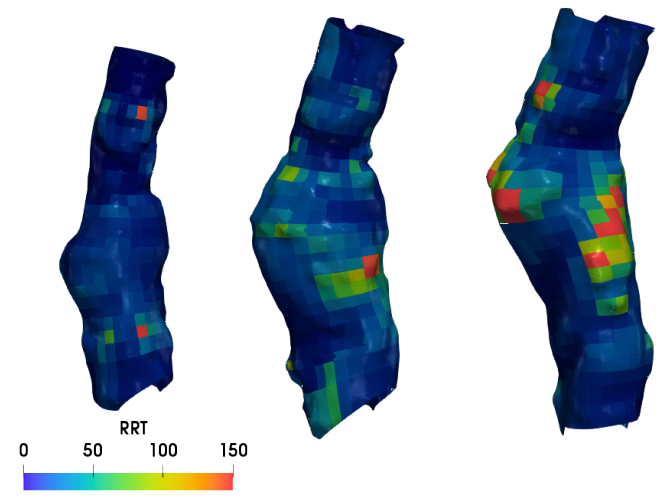

(a)

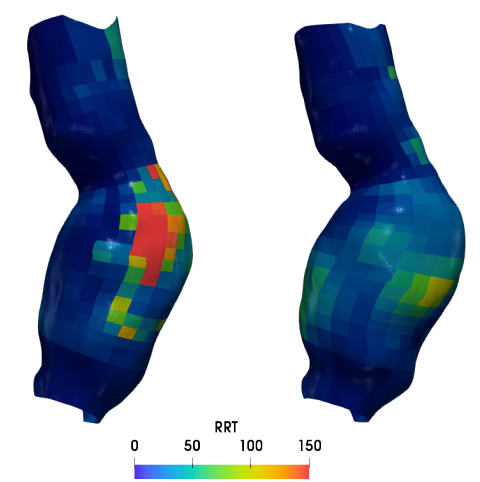

(c)

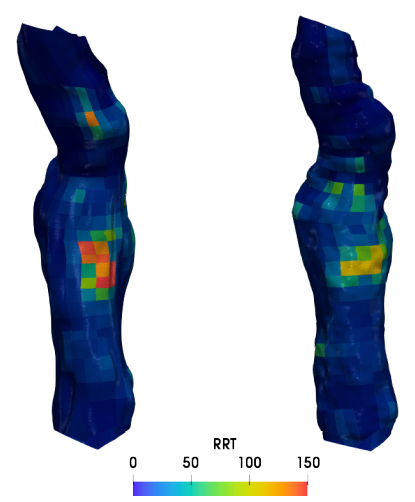

(b)
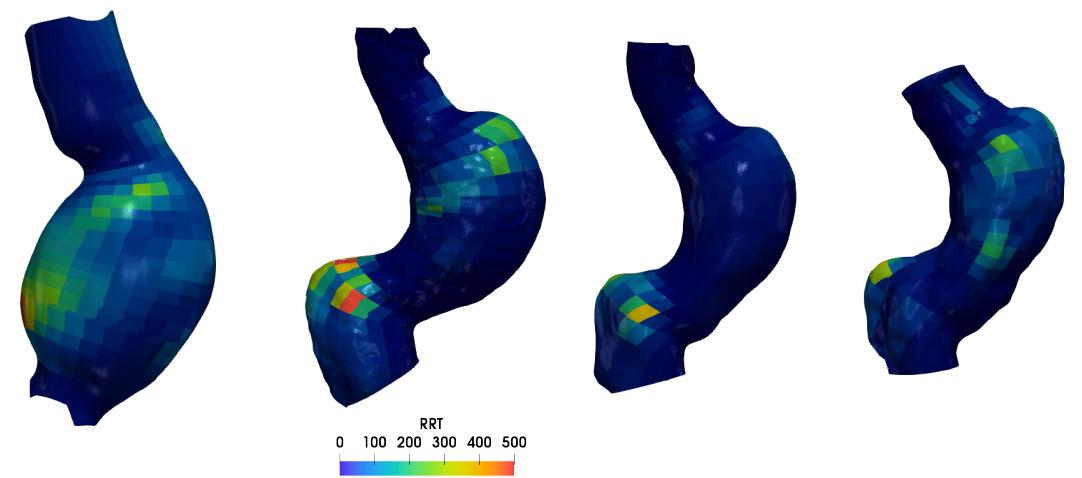

(d)

Figure V: For 4 patients, RRT averaged on patches. Patient 10 (a), exhibits a steady growth with time. Patient 28 (b): RRT standard deviation is relatively small compared to patient 29 (c). For patient 15 (d), RRT decreases before increasing. 


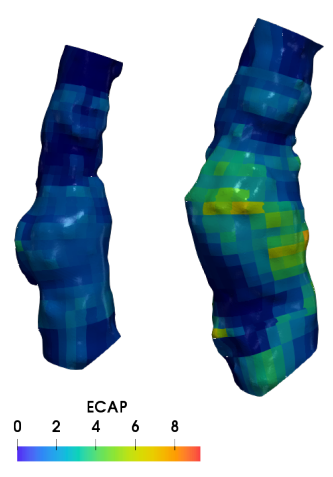

(a)

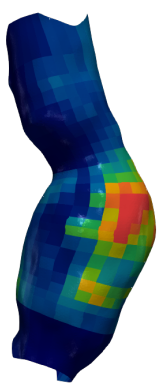

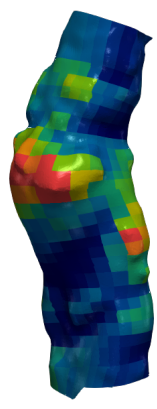

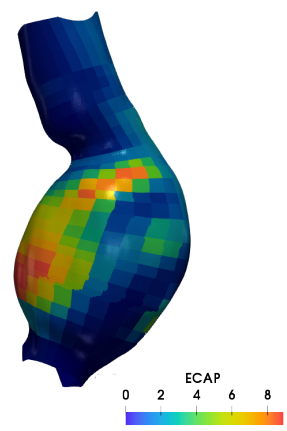

(c)

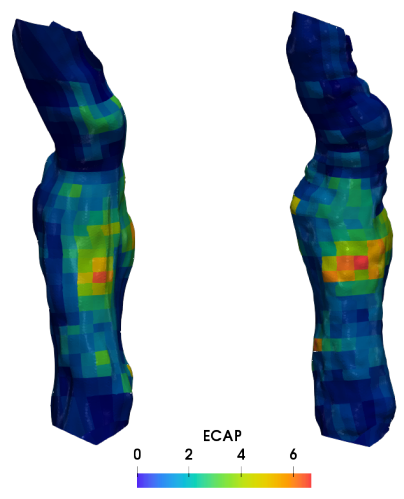

(b)

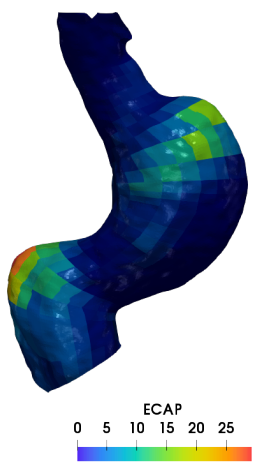

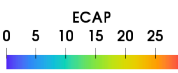
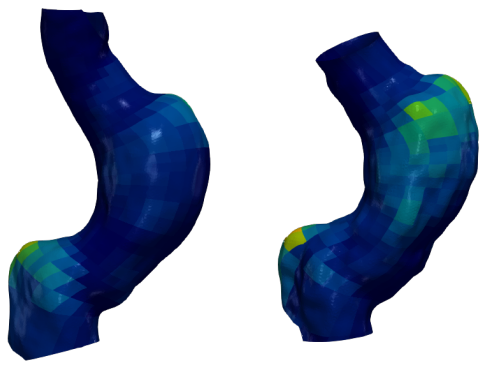

(d)

Figure VI: ECAP averaged on patches, for 4 patients. ECAP, similarly to the RRT contains information from OSI and TAWSS. Patient 10 (a), monotonic growth, patient 28 (b) with a small standard deviation compared to patient 29 (c). Patient 15 (d) ECAP average, decreases and then increases. 


\section{List of Tables}

1 Percentage of the 42 patients for which clinical data are available, per variable. Age, BMI (body mass index), $p_{\text {sys }}$ (systolic pressure) and $p_{\text {dias }}$ (diastolic pressure) are continuous variable and Sex, Dyslipidemia (DLP) and statins are discrete. . . . . . . . . . . . . . . . . . .

2 Statistical distribution of the clinical variables among the three groups. When a significant difference was observed $(p<0.05)$ between two groups, it was reported in the $4^{\text {th }}$ column. H-LR means a statistical difference between the High and Low-Risk groups, H-HR between the Healthy and High-Risk groups and LR-HR between the Low and High-Risk groups. Standard deviations are given in parentheses. . . . . . . . . . . . . . . . . .

3 Statistical distribution of the morphological variables among the three groups. When a significant difference was observed $(p<0.05)$ between two groups, it was reported in the $4^{\text {th }}$ column. H-LR means a statistical difference between the High and Low-Risk groups, H-HR between the Healthy and High-Risk groups and LR-HR between the Low and High-Risk groups. Standard deviations are given in parentheses. . . . . . . . . . . . . . . . .

4 Statistical distribution of the hemodynamic variables among the three groups. When a significant difference was observed $(p<0.05)$ between two groups, it was reported in the $4^{\text {th }}$ column. H-LR means a statistical difference between the High and Low-Risk groups, H-HR between the Healthy and High-Risk groups and LR-HR between the Low and High-Risk groups. Standard deviations are given in parentheses. . . . . . . . . . . . . . . . . . . .

$5 \quad$ Statistical distribution of the local annual variation of ILT thickness among the low and high-risk groups. When a significant difference was observed $(p<0.05)$ between two groups, it was reported in the $4^{\text {th }}$ column. H-LR means a statistical difference between the High and Low-Risk groups, H-HR between the Healthy and High-Risk groups and LR-HR between the Low and High-Risk groups. Standard deviations are given in parentheses. . . . . . . .

6 Statistical distribution of the local hemodynamic variables among the low and high-risk groups. When a significant difference was observed $(p<0.05)$ between two groups, it was reported in the $4^{\text {th }}$ column. LR-HR means a statistical difference between the Low and High-Risk groups. Standard deviations are given in parentheses. . . . . . . . . . . . . . . . . . . . . 55

7 p-values between AUCs from Figure 8 according to Delong et al. ${ }^{21}$ method. Significant values $(\leq 0.05)$ are in orange cells. . . . . . . . . . . . . 56

I Proximal resistance, compliance and distal resistance for the 0D-RCR model, from Xiao et al. $\frac{68}{}$ (in [CGS] units). . . . . . . . . . . . . . . 57

II Description of the various metrics used in the article. . . . . . . . . . 58 


\begin{tabular}{l|l} 
Clinical metric & Availability among patients (\%) \\
\hline Age & 71.4 \\
Sex & 100 \\
BMI & 40.5 \\
$p_{\text {sys }}$ & 61.9 \\
$p_{\text {dias }}$ & 61.9 \\
Dyslipidemia & 69.1 \\
Statins & 69.1
\end{tabular}

Table 1: Percentage of the 42 patients for which clinical data are available, per variable. Age, $B M I$ (body mass index), $p_{\text {sys }}$ (systolic pressure) and $p_{\text {dias }}$ (diastolic pressure) are continuous variable and Sex, Dyslipidemia (DLP) and statins are discrete. 


\begin{tabular}{|l|l|l|l|l|}
\hline Clinical variables & Healthy & Low-risk & High-risk & Statistical significance \\
\hline Age (yr) & $60.40(12.44)$ & $73.66(7.03)$ & $73.88(7.85)$ & H-LR, H-HR \\
Systolic pressure (mmHg) & $129.25(16.89)$ & $119.80(11.54)$ & $130.73(17.24)$ & LR-HR \\
Diastolic pressure (mmHg) & $74.88(8.67)$ & $68.91(8.65)$ & $77.21(13.85)$ & LR-HR \\
Dyslipidemia (DLP) (\%) & $0.33(0.47)$ & $0.84(0.37)$ & $0.82(0.38)$ & H-LR, H-HR \\
BMI & $25.92(4.91)$ & $31.20(5.73)$ & $28.65(5.95)$ & \\
Statins (\%) & $0.33(0.47)$ & $0.82(0.38)$ & $0.80(0.40)$ & H-LR, H-HR \\
\hline
\end{tabular}

Table 2: Statistical distribution of the clinical variables among the three groups. When a significant difference was observed $(p<0.05)$ between two groups, it was reported in the $4^{\text {th }}$ column. H-LR means a statistical difference between the High and Low-Risk groups, H-HR between the Healthy and High-Risk groups and LR-HR between the Low and High-Risk groups. Standard deviations are given in parentheses. 


\begin{tabular}{|c|c|c|c|c|}
\hline Morphological variables & Healthy & Low-risk & High-risk & Statistical significance \\
\hline Lumen surface area $\left(\mathrm{cm}^{2}\right)$ & $60.32(16.93)$ & $98.74(23.03)$ & $120.90(27.10)$ & H-LR, H-HR, LR-HR \\
\hline Lumen surface area, annual $\left(\mathrm{cm}^{2} \mathrm{yr}^{-1}\right)$ & - & $6.51(13.99)$ & $-2.14(81.85)$ & \\
\hline Lumen volume $(\mathrm{cm})$ & $24.95(11.58)$ & $57.17(20.75)$ & $79.62(34.28)$ & H-LR, H-HR, LR-HR \\
\hline Lumen volume, annual $\left(\mathrm{cm}^{3} \mathrm{yr}^{-1}\right)$ & - & $6.34(16.24)$ & $7.43(80.96)$ & \\
\hline ILT volume $(\mathrm{cm})$ & $0.00(0.00)$ & $38.99(30.80)$ & $65.90(44.25)$ & H-LR, H-HR, LR-HR \\
\hline ILT volume, annual $\left(\mathrm{cm}^{3} \mathrm{yr}^{-1}\right)$ & - & $3.28(22.55)$ & $33.95(154.98)$ & \\
\hline Total volume $(\mathrm{cm})$ & $24.95(11.58)$ & $96.17(41.25)$ & $145.52(53.53)$ & H-LR, H-HR, LR-HR \\
\hline Total volume, annual $\left(\mathrm{cm}^{3} \mathrm{yr}^{-1}\right)$ & - & $9.38(14.77)$ & $29.43(85.82)$ & \\
\hline$D_{\max }^{\text {lumen }}(\mathrm{mm})$ & $18.18(3.52)$ & $28.89(5.74)$ & $32.94(8.04)$ & H-LR, H-HR, LR-HR \\
\hline$D_{\max }^{\text {lumen }}$, annual $\left(\mathrm{mmyr}^{-1}\right)$ & - & $1.07(3.85)$ & $2.81(10.72)$ & \\
\hline$D_{\max }(\mathrm{mm})$ & $18.18(3.52)$ & $43.72(7.37)$ & $54.40(8.77)$ & H-LR, H-HR, LR-HR \\
\hline$D_{\max }$, annual $\left(\operatorname{mm~yr}^{-1}\right)$ & - & $1.14(3.31)$ & $3.61(4.55)$ & LR-HR \\
\hline Lumen NSI (-) & $1.22(0.02)$ & $1.18(0.04)$ & $1.18(0.05)$ & H-LR, H-HR \\
\hline ILT coverage $(\%)$ & - & $64.63(24.92)$ & $67.89(19.09)$ & \\
\hline ILT coverage, annual $\left(\mathrm{yr}^{-1}\right)$ & - & $-2.07(13.06)$ & $33.92(237.39)$ & \\
\hline$H_{\max }^{I L T}(\mathrm{~mm})$ & - & $7.89(4.12)$ & $11.65(5.31)$ & LR-HR \\
\hline$H_{m i n}^{I L T}(\mathrm{~mm})$ & - & $0.31(0.65)$ & $0.17(0.46)$ & \\
\hline$H_{\text {mean }}^{I L T}(\mathrm{~mm})$ & - & $3.09(1.80)$ & $4.05(2.34)$ & LR-HR \\
\hline$H_{\text {stdev }}^{I L T}(\mathrm{~mm})$ & - & $2.55(1.36)$ & $3.71(1.64)$ & LR-HR \\
\hline
\end{tabular}

Table 3: Statistical distribution of the morphological variables among the three groups. When a significant difference was observed $(p<0.05)$ between two groups, it was reported in the $4^{\text {th }}$ column. H-LR means a statistical difference between the High and Low-Risk groups, H-HR between the Healthy and High-Risk groups and LR-HR between the Low and High-Risk groups. Standard deviations are given in parentheses. 


\begin{tabular}{|c|c|c|c|c|}
\hline hemodynamic variables & Healthy & Low-risk & High-risk & Statistical significance \\
\hline$O S I_{\max }(-)$ & $0.36(0.04)$ & $0.38(0.04)$ & $0.37(0.05)$ & \\
\hline$O S I_{\min }(-)$ & $0.02(0.02)$ & $0.02(0.02)$ & $0.02(0.03)$ & \\
\hline$O S I_{\text {mean }}(-)$ & $0.16(0.04)$ & $0.18(0.04)$ & $0.17(0.04)$ & \\
\hline$O S I_{\text {stdev }}(-)$ & $0.11(0.01)$ & $0.11(0.01)$ & $0.11(0.02)$ & \\
\hline$T A W S S_{\max }(\mathrm{Pa})$ & $0.66(0.31)$ & $0.58(0.28)$ & $0.54(0.22)$ & \\
\hline$T A W S S_{\min }(\mathrm{Pa})$ & $0.23(0.08)$ & $0.11(0.06)$ & $0.09(0.05)$ & H-LR, H-HR, LR-HR \\
\hline$T A W S S_{\text {mean }}(\mathrm{Pa})$ & $0.40(0.17)$ & $0.27(0.11)$ & $0.23(0.10)$ & H-LR, H-HR \\
\hline$T A W S S_{\text {stdev }}(\mathrm{Pa})$ & $0.16(0.09)$ & $0.16(0.09)$ & $0.16(0.07)$ & \\
\hline$W S S G_{\max }\left(\mathrm{Pa} \mathrm{m}^{-1}\right)$ & $135.60(71.51)$ & $124.55(70.32)$ & $116.32(61.23)$ & \\
\hline$W S S G_{\min }\left(\mathrm{Pa} \mathrm{m}^{-1}\right)$ & $17.42(9.48)$ & $9.30(7.30)$ & $6.79(7.10)$ & H-LR, H-HR \\
\hline$W S S G_{\text {mean }}\left(\mathrm{Pa} \mathrm{m}^{-1}\right)$ & $61.03(32.52)$ & $44.74(25.82)$ & $38.89(23.32)$ & \\
\hline$W S S G_{\text {stdev }}\left(\mathrm{Pa} \mathrm{m}^{-1}\right)$ & $61.04(44.25)$ & $41.97(24.82)$ & $45.17(25.02)$ & \\
\hline$R R T_{\max }\left(\mathrm{Pa}^{-1}\right)$ & $22.64(17.25)$ & $48.26(30.80)$ & $58.15(34.73)$ & H-LR, H-HR \\
\hline$R R T_{\min }\left(\mathrm{Pa}^{-1}\right)$ & $2.34(1.33)$ & $2.63(1.32)$ & $3.12(1.84)$ & \\
\hline$R R T_{\text {mean }}\left(\mathrm{Pa}^{-1}\right)$ & $8.20(6.17)$ & $15.68(8.01)$ & $19.00(10.00)$ & H-LR, H-HR, LR-HR \\
\hline$R R T_{\text {stdev }}\left(\mathrm{Pa}^{-1}\right)$ & $8.20(6.51)$ & $21.68(16.56)$ & $24.22(15.69)$ & H-LR, H-HR \\
\hline$E C A P_{\max }\left(\mathrm{Pa}^{-1}\right)$ & $0.66(0.39)$ & $1.42(0.76)$ & $1.77(0.92)$ & H-LR, H-HR, LR-HR \\
\hline$E C A P_{\min }\left(\mathrm{Pa}^{-1}\right)$ & $0.03(0.03)$ & $0.02(0.03)$ & $0.04(0.06)$ & \\
\hline$E C A P_{\text {mean }}\left(\mathrm{Pa}^{-1}\right)$ & $0.26(0.19)$ & $0.48(0.22)$ & $0.59(0.32)$ & H-LR, H-HR, LR-HR \\
\hline$E C A P_{\text {stdev }}\left(\mathrm{Pa}^{-1}\right)$ & $0.20(0.11)$ & $0.48(0.27)$ & $0.59(0.31)$ & H-LR, H-HR, LR-HR \\
\hline
\end{tabular}

Table 4: Statistical distribution of the hemodynamic variables among the three groups. When a significant difference was observed $(p<0.05)$ between two groups, it was reported in the $4^{\text {th }}$ column. H-LR means a statistical difference between the High and Low-Risk groups, H-HR between the Healthy and High-Risk groups and LR-HR between the Low and High-Risk groups. Standard deviations are given in parentheses. 


\begin{tabular}{|l|l|l|l|}
\hline Local morphological variables & Low-risk & High-risk & Statistical significance \\
\hline local change of $H_{\max }^{I L T}\left(\mathrm{~mm} \mathrm{yr}^{-1}\right)$ & $4.55(4.27)$ & $7.31(4.58)$ & LR-HR \\
local change of $H_{\text {min }}^{I L T}\left(\mathrm{~mm} \mathrm{yr}^{-1}\right)$ & $-4.96(6.47)$ & $-16.78(33.77)$ & LR-HR \\
local change of $H_{\text {mean }}^{I L T}\left(\mathrm{~mm} \mathrm{yr}^{-1}\right)$ & $-0.11(1.60)$ & $-1.18(5.38)$ & \\
\hline
\end{tabular}

Table 5: Statistical distribution of the local annual variation of ILT thickness among the low and high-risk groups. When a significant difference was observed $(p<0.05)$ between two groups, it was reported in the $4^{\text {th }}$ column. H-LR means a statistical difference between the High and Low-Risk groups, H-HR between the Healthy and High-Risk groups and LR-HR between the Low and High-Risk groups. Standard deviations are given in parentheses. 


\begin{tabular}{|l|l|l|l|}
\hline Local hemodynamic variables & Low-risk & High-risk & Statistical significance \\
\hline local change of $O S I_{\max }\left(\mathrm{yr}^{-1}\right)$ & $0.29(0.41)$ & $1.26(3.70)$ & \\
local change of $O S I_{\text {min }}\left(\mathrm{yr}^{-1}\right)$ & $-0.29(0.38)$ & $-1.14(2.67)$ & LR-HR \\
local change of $O S I_{\text {mean }}\left(\mathrm{yr}^{-1}\right)$ & $0.00(0.06)$ & $0.05(0.47)$ & \\
local change of $T A W S S_{\max }\left(\mathrm{Payr}^{-1}\right)$ & $0.27(0.36)$ & $1.08(3.50)$ & \\
local change of $T A W S S_{\text {min }}\left(\mathrm{Pa} \mathrm{yr}^{-1}\right)$ & $-0.42(0.70)$ & $-1.47(3.86)$ & LR-HR \\
local change of $T A W S S_{\text {mean }}\left(\mathrm{Pa} \mathrm{yr}^{-1}\right)$ & $-0.02(0.10)$ & $-0.04(0.25)$ & \\
local change of $W S S G_{\text {max }}\left(\mathrm{Pam}^{-1} \mathrm{yr}^{-1}\right)$ & $81.56(108.31)$ & $287.46(963.86)$ & \\
local change of $W S S G_{\min }\left(\mathrm{Pa} \mathrm{m}^{-1} \mathrm{yr}^{-1}\right)$ & $-111.53(176.19)$ & $-350.49(829.66)$ & LR-HR \\
local change of $\left.W S S G_{\text {mean }}\left(\mathrm{Pa} \mathrm{m}^{-1} \mathrm{yr}^{-1}\right)\right)$ & $-3.86(24.29)$ & $4.27(109.43)$ & \\
local change of $R R T_{\text {max }}\left(\mathrm{Pa}^{-1} \mathrm{yr}^{-1}\right)$ & $44.64(73.56)$ & $245.22(658.03)$ & LR-HR \\
local change of $R R T_{\text {min }}\left(\mathrm{Pa}^{-1} \mathrm{yr}^{-1}\right)$ & $-44.47(90.49)$ & $-225.57(538.46)$ & LR-HR \\
local change of $R R T_{\text {mean }}\left(\mathrm{Pa}^{-1} \mathrm{yr}^{-1}\right)$ & $0.64(6.76)$ & $8.16(55.48)$ & \\
local change of $E C A P_{\text {max }}\left(\mathrm{Pa}^{-1} \mathrm{yr}^{-1}\right)$ & $4.61(7.11)$ & $23.63(58.92)$ & LR-HR \\
local change of $E C A P_{\text {min }}\left(\mathrm{Pa}^{-1} \mathrm{yr}^{-1}\right)$ & $-0.44(0.56)$ & $-1.85(3.48)$ & LR-HR \\
local change of $E C A P_{\text {mean }}\left(\mathrm{Pa}^{-1} \mathrm{yr}^{-1}\right)$ & $1.31(2.35)$ & $7.39(21.40)$ & \\
\hline
\end{tabular}

Table 6: Statistical distribution of the local hemodynamic variables among the low and highrisk groups. When a significant difference was observed $(p<0.05)$ between two groups, it was reported in the $4^{\text {th }}$ column. LR-HR means a statistical difference between the Low and High-Risk groups. Standard deviations are given in parentheses. 


\begin{tabular}{|c|c|c|c|c|c|}
\cline { 2 - 6 } \multicolumn{1}{c|}{} & Dmax & Clinical & Morpho. & Flow & All \\
\hline Dmax & & 0.393 & 0.008 & 0.006 & 0.006 \\
\hline Clinical & & & 0.004 & 0.004 & 0.004 \\
\hline Morpho. & & & & 0.561 & 0.207 \\
\hline Hemo. & & & & & 0.281 \\
\hline All & & & & & \\
\hline
\end{tabular}

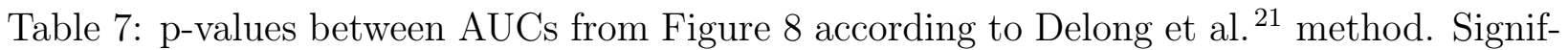
icant values $(\leq 0.05)$ are in orange cells. 


\begin{tabular}{|l|l|l|l|}
\hline Outlets & Rp & C & Rd \\
\hline Mes. Sup. & $6.7 * 10^{3}$ & $8.11 * 10^{6}$ & $1.13 * 10^{5}$ \\
\hline Celiac & $6.7 * 10^{3}$ & $8.11 * 10^{6}$ & $1.13 * 10^{5}$ \\
\hline Renal & $1.2 * 10^{4}$ & $1.8 * 10^{-5}$ & $4.8 * 10^{4}$ \\
\hline Int. Iliac & $4.55 * 10^{3}$ & $1.582 * 10^{-5}$ & $7.7 * 10^{4}$ \\
\hline Ext. Iliac & $4.8 * 10^{3}$ & $1.75 * 10^{-5}$ & $8.2 * 10^{4}$ \\
\hline
\end{tabular}

Table I: Proximal resistance, compliance and distal resistance for the 0D-RCR model, from

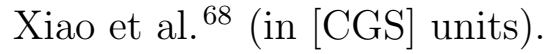




\begin{tabular}{|c|c|c|c|}
\hline & $\begin{array}{l}\text { Metric } \\
\text { notation }\end{array}$ & Extraction & Remarks \& litt. \\
\hline \multirow{9}{*}{ 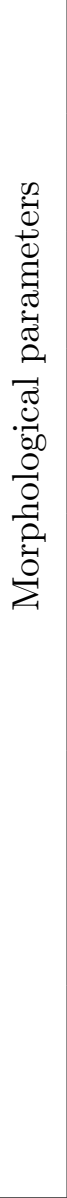 } & $D_{\max }^{\text {lumen }}$ & $\begin{array}{l}\text { maximal diameter of the lumen in a plane } \\
\text { orthogonal to the luminal centerline }\end{array}$ & - \\
\hline & $D_{\max }$ & $\begin{array}{l}\text { maximal diameter of the AAA (inc. ILT) in a } \\
\text { plane orthogonal to the luminal centerline }\end{array}$ & $\begin{array}{l}\text { Current clinical } \\
\text { criteria. }\end{array}$ \\
\hline & $H^{I L T}$ & $\begin{array}{l}\text { thrombus thickness, computed as the Euclidean } \\
\text { distance between the lumen and ILT }\end{array}$ & - \\
\hline & $\begin{array}{l}\text { Lumen } \\
\text { centerline } \\
\text { curvature }\end{array}$ & inverse of the radius of the local oscillating circle & Shum et al. $\underline{55}$ \\
\hline & $\begin{array}{l}\text { Lumen } \\
\text { centerline } \\
\text { tortuosity }\end{array}$ & $\begin{array}{l}\text { ratio between the centerline length and the } \\
\text { endpoints distance. }\end{array}$ & Shum et al. $\stackrel{55}{55}$ \\
\hline & Lumen NSI & $\frac{1}{2.199} \frac{\sqrt{\text { Area }}}{\sqrt[3]{\text { Volume }}}$ & Raghavan et al. $\underline{49}$ \\
\hline & $\begin{array}{l}\text { Lumen (ILT) } \\
\text { volume }\end{array}$ & $\begin{array}{l}\text { volume of the lumen (ILT) between the renal } \\
\text { and the iliac bifurcation. }\end{array}$ & - \\
\hline & $\begin{array}{l}\text { Lumen (ILT) } \\
\text { surface area }\end{array}$ & $\begin{array}{l}\text { surface of the lumen (ILT) between the renal } \\
\text { and the iliac bifurcation. }\end{array}$ & - \\
\hline & ILT coverage & $\begin{array}{l}\text { percentage of the lumen covered with thrombus. } \\
\text { The observed quantity is the ratio of lumen } \\
\text { outer wall area exposed to ILT to the total area, } \\
\text { not the aortic wall covered in ILT }\end{array}$ & - \\
\hline \multirow{5}{*}{ 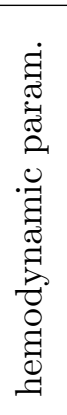 } & TAWSS & $\frac{1}{T} \int_{0}^{T}\left|\tau_{W}\right| \mathrm{dt}$ & $\begin{array}{l}\text { Bluestein et al. } .9 \\
\text { and Arzani et al. } . \underline{4}\end{array}$ \\
\hline & OSI & $\frac{1}{2}\left(1-\frac{\left|\int_{0}^{T} \tau_{W} \mathrm{dt}\right|}{\int_{0}^{T}\left|\tau_{W}\right| \mathrm{dt}}\right)$ & Arzani al. ${ }^{4}$ \\
\hline & RRT & $\frac{1}{(1-2 O S I) T A W S S}$ & Himburg et al. .36 \\
\hline & ECAP & $\frac{O S I}{T A W S S}$ & Di Achille et al. ${ }^{24}$ \\
\hline & WSSG & $|\nabla W S S|$ & Nagel et al. $\underline{45}$ \\
\hline
\end{tabular}

Table II: Description of the various metrics used in the article. 